OPEN ACCESS

Edited by:

Carlos Gómez,

University of Valladolid, Spain

Reviewed by:

Yang Wang,

Medical College of Wisconsin

United States

Arun Bokde,

Trinity College Dublin, Ireland

*Correspondence:

Bin Wang

wangbin01@tyut.edu.cn

Jie Xiang

xiangjie@tyut.edu.cn

Specialty section:

This article was submitted to

Applied Neuroimaging,

a section of the journal

Frontiers in Neurology

Received: 24 May 2019

Accepted: 14 January 2020

Published: 05 February 2020

Citation:

Cao R, Wang X, Gao Y, Li T, Zhang H,

Hussain W, Xie $Y$, Wang J, Wang $B$

and Xiang J (2020) Abnormal

Anatomical Rich-Club Organization

and Structural-Functional Coupling in

Mild Cognitive Impairment and

Alzheimer's Disease.

Front. Neurol. 11:53.

doi: 10.3389/fneur.2020.00053

\section{Abnormal Anatomical Rich-Club Organization and Structural-Functional Coupling in Mild Cognitive Impairment and Alzheimer's Disease}

\author{
Rui Cao ${ }^{1}$, Xin Wang ${ }^{2}$, Yuan $\mathrm{Gao}^{2}$, Ting $\mathrm{Li}^{3}$, Hui Zhang ${ }^{4}$, Waqar Hussain ${ }^{2}$, Yunyan Xie ${ }^{5}$, \\ Jing Wang ${ }^{6}$, Bin Wang ${ }^{2 *}$ and Jie Xiang ${ }^{2 *}$ \\ ${ }^{1}$ College of Software, Taiyuan University of Technology, Taiyuan, China, ${ }^{2}$ College of Information and Computer, Taiyuan \\ University of Technology, Taiyuan, China, ${ }^{3}$ School of Life Science, Beijing Institute of Technology, Beijing, China, ${ }^{4}$ Department \\ of Radiology, First Hospital of Shanxi Medical University, Taiyuan, China, ${ }^{5}$ Department of Neurology, Xuanwu Hospital, Capital \\ Medical University, Beijing, China, ${ }^{6}$ Department of Health management, Aerospace Center Hospital, Peking University \\ Aerospace School of Clinical Medicine, Beijing, China
}

Emerging research indicates interruptions in the wiring organization of the brain network in Mild cognitive impairment (MCl) and Alzheimer's disease (AD). Due to the important role of rich-club organization in distinguishing abnormalities of $A D$ patients and the close relationship between structural connectivity (SC) and functional connectivity (FC), our study examined whether changes in SC-FC coupling and the relationship with abnormal rich-club organizations during the development of diseases may contribute to the pathophysiology of AD. Structural diffusion-tensor imaging (DTI) and resting-state functional magnetic resonance imaging (fMRI) were performed in 38 normal controls (NCs), $40 \mathrm{MCl}$ patients and $19 \mathrm{AD}$ patients. Measures of the rich-club structure and its role in global structural-functional coupling were administered. Our study found decreased levels of feeder and local connectivity in $\mathrm{MCl}$ and $\mathrm{AD}$ patients, which were the main contributing factors to the lower efficiency of the brain structural network. Another important finding was that we have more accurately characterized the changing pattern of functional brain dynamics. The enhanced coupling between SC and $\mathrm{FC}$ in $\mathrm{MCl}$ and $\mathrm{AD}$ patients might be due to disruptions in optimal structural organization. More interestingly, we also found increases in the SC-FC coupling for feeder and local connections in $\mathrm{MCl}$ and $\mathrm{AD}$ patients. SC-FC coupling also showed significant differences between $\mathrm{MCl}$ and $A D$ patients, mainly between the abnormal feeder connections. The connection density and coupling strength were significantly correlated with clinical metrics in patients. The present findings enhanced our understanding of the neurophysiologic mechanisms associated with $\mathrm{MCl}$ and $\mathrm{AD}$.

Keywords: Alzheimer's disease, mild cognitive impairment, rich-club organization, SC-FC coupling, brain network 


\section{INTRODUCTION}

The human brain is a complex network that supports efficient communication through structurally and functionally interconnected brain units (1). The motivation for studying the brain's underlying connectivity is the theory that the function of the brain depends on the network organization of the whole brain rather than individual nodes or individual connections (2-4). Generally, the brain connectome has been directly probed by structural connectivity (SC) derived from diffusion-tensor imaging (DTI), which represents anatomical wiring diagrams, and functional connectivity (FC) derived from resting-state functional magnetic resonance imaging (fMRI), which reflects the synchronization of neuronal activities in different brain regions $(5,6)$. Recently, researchers have argued that the functional network connections of the human brain are limited by the potential anatomical white matter pathway $(5,7-17)$. The association between SC and FC, called structuralfunctional (SC-FC) coupling, and the joint study of SC and FC can describe the functional dynamics of the brain from a structural topological perspective and may detect subtle brain changes more sensitively than any single imaging modality ( 7 , 18, 19). A large number of studies have demonstrated that the abnormal functional dynamics in brain network dysfunction in schizophrenia, idiopathic generalized epilepsy, migraine, and Alzheimer's disease were caused by abnormalities due to brain diseases $(1,19-21)$.

Alzheimer's disease (AD) is a neurodegenerative disease that is clinically characterized by progressive memory impairment and loss of cognitive functions and is considered a syndrome of disconnectivity among brain regions (22). Mild cognitive impairment $(\mathrm{MCI})$ is a prodromal stage of $\mathrm{AD}$, with a conversion rate of $10-15 \%$ to $A D$ annually (23). Studies using neuroimaging techniques, such as DTI and fMRI, suggested the destruction of white matter pathways or SC among the pathological regions attributed to abnormalities in FC and cognitive decline in $A D$ patients (24). A large number of studies with $A D$ patients showed SC and/or FC abnormalities among related brain regions, such as the hippocampus and precuneus (14, 25, 26). Dai et al. (21) demonstrated overlapping and distinct network disruptions in SC and $\mathrm{FC}$ in $\mathrm{AD}$. By analyzing the functional dynamics of the brain from a structural topological perspective, Su et al. (11) observed a decrease in the strength of SC-FC coupling and speculated that decreased coupling may be suggestive of less dynamic and more stringent brain function in $\mathrm{AD}$ patients. Wang et al. (27) argued that the disruption of optimal structural organization may have given rise to alterations in functional dynamics. Recently, Dai et al. (21) reported that the increased functional dynamics of the default-mode network in $\mathrm{AD}$ patients indicated that $\mathrm{AD}$ leads to a strengthened relationship between FC and the underlying anatomical connectivity. More importantly, the disruptions in structural-functional relationships in patients with $\mathrm{AD}$ might be the primary cause of the cognitive deficits (28).

Graph theory analyses have shown changes in the topological properties of brain networks in $\mathrm{AD}$, including decreases in global and local efficiency and impairments in small-world properties
$(21,26,29,30)$. Rich-club organization is characterized by a tendency for hub regions to be more densely connected among themselves than with peripheral regions (31). As high-capacity centrical cores, the rich-club connections play a central part in the global neural information communication of the brain $(32,33)$. Previous network studies have reported overall level interruptions in the brain network of SC and FC in patients, together with significant lesions distributed throughout the hub regions in the frontal, temporal, and parietal cortices (34-36). A study by Mallio et al. (37) reported a trend of epicentral disruption around the entorhinal and hippocampal regions in $\mathrm{MCI}$ and $\mathrm{AD}$ patients, which is consistent with the transneuronal spread hypothesis. Daianu et al. (36) suggested that structural network disruptions predominated in more remotely connected regions in patients. In our previous studies, the disturbances in rich-club organization dynamically and potently disrupted connectivities among peripheral regions in preclinical $\mathrm{AD}$ and MCI patients, and the disrupted connectivities spread to the rich-club regions in the brains of patients with $\operatorname{AD}(26,38)$. Compared with the rich-club connections, the feeder and local connections are demonstrated to be impaired earlier and more severely (36). Recently, Dai et al. (21) found that the rich-club connections had significantly increased SC-FC coupling in AD. But only $\mathrm{AD}$ patients were studied, the progressive changes at the early prodromal stage of neurodegenerative diseases were still unclear. Wang et al. (27) found that moderate CIND had higher SC-FC correlation than HC, while Sun et al. (11) observed a decrease in the strength of SC-FC coupling in $\mathrm{AD}$ patients. However, the results of the previous work are inconsistent, and the relationship between abnormal anatomical rich-club organization and disruptions in structural-functional relationships in $\mathrm{MCI}$ and $\mathrm{AD}$ patients remains unclear.

In this study, we examined the underlying abnormal anatomical rich-club organization using DTI and resting-state fMRI data from a group of 38 normal controls (NCs), 40 MCI patients and $19 \mathrm{AD}$ patients. We anticipated that the functional dynamics of patients would change due to these disproportionately disrupted white matter connections during the progression of $\mathrm{AD}$. Finally, the clinical relevance of the structural rich-club organization and SC-FC coupling was also examined in patients to promote a mechanistic understanding of the dynamic changes in clinical manifestations and identify a potential biomarker that can detect subtle brain connectivity disruption with high sensitivity.

\section{MATERIALS AND METHODS}

\section{Participants}

A total of 97 participants were collected from the Alzheimer's Disease Neuroimaging Initiative (ADNI) database (http://adni. loni.ucla.edu), including 19 patients with $\mathrm{AD}$ (8 females), 40 patients with MCI (20 females), and 38 normal control subjects (19 females) (Table 1). The ADNI was approved by the ethics committee of the National Institute on Aging, the National Institute of Biomedical Imaging and Bioengineering, and the local ethics committee of each participating site. The participants also provided written informed consent when they registered for 
TABLE 1 | Demographic characteristics and neuropsychological test results.

\begin{tabular}{|c|c|c|c|c|c|}
\hline Characteristic & NC $(n=38)$ & $\operatorname{MCl}(n=40)$ & $\mathrm{AD}(n=19)$ & Test statistic & $P$-value \\
\hline Age (y) & $75.1 \pm 1.6$ & $75.3 \pm 1.1$ & $74.6 \pm 2.3$ & $F=0.04$ & 0.96 \\
\hline Sex (male/female) & $19 / 19$ & $20 / 20$ & $11 / 8$ & $x 2=0.381$ & 0.826 \\
\hline Education level (y) & $17.0 \pm 0.4$ & $15.6 \pm 0.5$ & $15.4 \pm 0.5$ & $F=3.55$ & $0.033^{\mathrm{ab}}$ \\
\hline CDR & $0.03 \pm 0.02$ & $0.49 \pm 0.07$ & $1.00 \pm 0.11$ & $F=54.59$ & $<0.001^{\mathrm{abc}}$ \\
\hline FAQ & $0.08 \pm 0.06$ & $3.10 \pm 0.77$ & $15.06 \pm 2.04$ & $F=39.33$ & $<0.001^{\mathrm{abc}}$ \\
\hline MMSE & $29.24 \pm 0.17$ & $27.90 \pm 0.29$ & $21.53 \pm 1.08$ & $F=73.98$ & $<0.001^{a b c}$ \\
\hline MoCA & $25.00 \pm 0.39$ & $20.05 \pm 0.91$ & $13.87 \pm 1.35$ & $F=48.68$ & $<0.001^{\mathrm{abc}}$ \\
\hline GDS & $0.50 \pm 0.12$ & $1.83 \pm 0.25$ & $1.84 \pm 0.32$ & $F=12.84$ & $<0.001^{\mathrm{ab}}$ \\
\hline
\end{tabular}

${ }^{A}$ Values are given as the mean $\pm S D$.

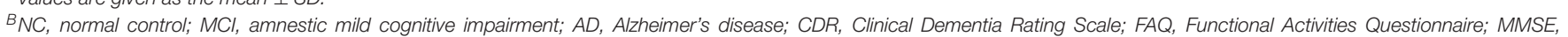
Mini-Mental State Examination; MoCA, Montreal Cognitive Assessment; GDS, Geriatric Depression Scale.

${ }^{a} \mathrm{NC}$ group and $\mathrm{MCl}$ patients showed significant differences $(P<0.05)$.

${ }^{b} \mathrm{NC}$ group and $A D$ patients showed significant differences $(P<0.05)$.

${ }^{c} \mathrm{MCl}$ patients and $A D$ patients showed significant differences $(P<0.05)$.

imaging and completed the questionnaires. All participants were assessed by a standardized clinical evaluation protocol, including a neurologic examination, a medical history interview, and a battery of neuropsychological tests (26). All participants were right-handed and had no history of neurological or psychiatric disorders. The neuropsychological tests included the Clinical Dementia Rating Scale (CDR) (39), Geriatric Depression Scale (GDS) (40), Functional Assessment Questionnaire (FAQ) (41), Mini-Mental State Examination (MMSE) (42), and Montreal Cognitive Assessment (MoCA) (43). The NCs with CDR scores of 0 and MMSE scores of 24-30 were classified as non-demented, non-MCI, and non-depressed. The MCI patients had CDR scores of 0.5 and MMSE scores of 23-30. The AD patients met the National Institute of Neurological and Communicative Disorders and Stroke and the Alzheimer's Disease and Related Disorders Association (NINCDS/ADRDA) criteria for probable AD and had CDR scores of 0.5 or 1 and MMSE scores of 14-26.

\section{Imaging}

Brain imaging was performed for all participants with a 3-T Siemens scanner according to the ADNI acquisition protocol. Structural DTI data, T1-weighted data and resting-state fMRI data were collected from all participants. During the data acquisition, headphones and cushions were used to minimize subject motion artifacts and scanner noise. The DTI data of each subject were collected three times using an echo planar imaging (EPI) sequence with the following parameters: 30 independent directions; slices $=31$; repetition time $(\mathrm{TR})=12400.0 \mathrm{~ms}$; echo time $(\mathrm{TE})=95.0 \mathrm{~ms}$; field of view $(\mathrm{FOV})=2088.0 \times$ $2088.0 \mathrm{~mm}^{2}$; flip angle $=90.0$ degrees; acquisition matrix $=1044$ $\times 1044$; and slice thickness $=2.0 \mathrm{~mm}$. The resting-state fMRI data included 197 functional volumes and were acquired with the following parameters: $\mathrm{TE}=30.0 \mathrm{~ms} ; \mathrm{TR}=3000.0 \mathrm{~ms}$; slice thickness $=3.4 \mathrm{~mm}$; flip angle $=90.0$ degrees; and 48 slices. During the data acquisition, all subjects were requested to relax their minds, open their eyes, and move as little as possible. T1-weighted MR images were obtained by a 3D magnetization-prepared rapid gradient echo (MPRAGE) using the following parameters: 176 sagittal slices; TR $=2300.0 \mathrm{~ms}$; slice thickness $=1.0 \mathrm{~mm}$; flip angle $=9.0$ degrees; $\mathrm{TE}=2.98 \mathrm{~ms}$; $\mathrm{FOV}=240 \times 256 \mathrm{~mm}^{2}$; and acquisition matrix $=240 \times 256$. All DTI data, T1-weighted data and resting-state fMRI data were checked for quality assurance to exclude scans with excessive motion and/or artifacts after preprocessing corrections; all scans were included.

\section{Image Preprocessing}

Anatomical parcellation: Freesurfer software was used to segment the T1 images of each subject. Freesurfer uses a surface-based alignment procedure, which might be more accurate than a volume-based alignment of a cortical atlas (44). Freesurfer applies a standardized processing pipeline to the T1 image, including skull stripping, volumetric labeling, intensity normalization, white matter segmentation, surface atlas registration, surface extraction, and gyral labeling. We used the Brainnetome atlas (BN atlas) and provided parcellation of 210 cortical and 36 subcortical subregions (45). The cortical and subcortical parcellation of each individual in native T1 space was transformed to the native DWI or fMRI space by applying a rigid body transformation computed with the FMRIB Software Library (FSL, http://fsl.fmrib.ox.ac.uk/fsl/fslwiki) FLIRT tool (46).

\section{DTI Preprocessing}

We used the Pipeline for Analyzing braiN Diffusion imAges toolbox (PANDA, http://www.nitrc.org/projects/panda) to preprocess DTI data following a method illustrated previously (47). PANDA is a MATLAB toolbox for pipeline processing of diffusion MRI images based on the Diffusion Toolkit (http:// www.trackvis.org/dtk/) and the FSL tool. First, we corrected for head motion and eddy current distortions. Subjects with more than $3 \mathrm{~mm}$ or degrees of head movement in any direction were removed. Then, we calculated the fractional anisotropy (FA) of each voxel and used the affine transformation to coregister FA image with the corresponding T1 image in the native space. After that, each region of the subject was defined as a brain network node. Reconstruction of WM pathways (referred to as fibers or tracts) for each individual DTI data using deterministic white matter tractography based on the fiber assignment by continuous tracking (FACT) algorithm $(48,49)$. Eight seeds followed the 


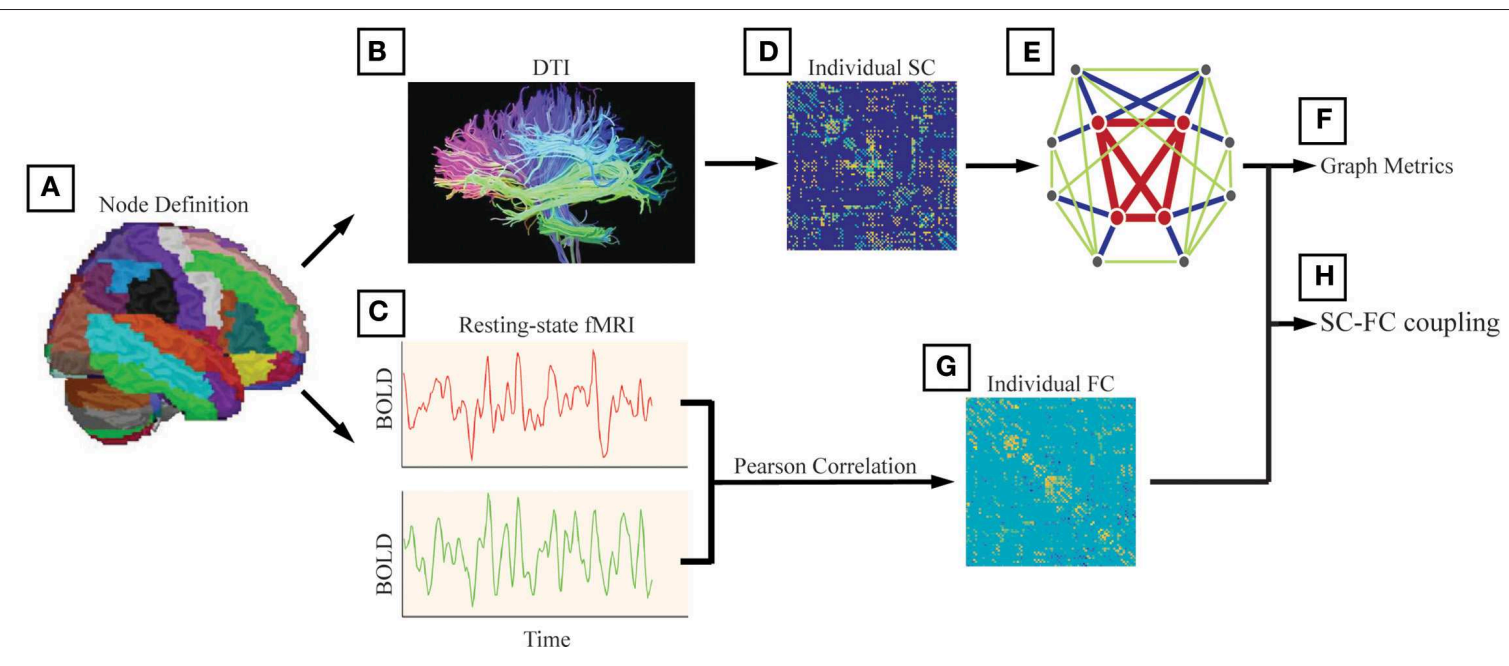

FIGURE 1 | The analysis steps were performed following Li et al. (20). (A) We used the Brainnetome atlas (BN atlas) and provided parcellation of 210 cortical and 36 subcortical subregions. We also used the automated anatomical labeling (AAL) atlas and this parcellation divided the cortical surface into 90 regions (45 per hemisphere). The method and results are shown in Supplementary. (B) Structural connectivity (SC) was defined as at least 3 bundles of fibers (>2) between any two regions. (C) Functional connectivity (FC) between any two nodes was computed as the Pearson correlation between the blood-oxygenlevel dependent (BOLD) time series. (D) The weighted networks were calculated as the number of fibers divided by the sum of the surface areas of the 2 interconnected ROls. (E) We generated a binary and undirected network for each participant with the structural connectivity between two regions set as 1 if the corresponding weight was positive (weight $>0$ ). (F) The investigated graph metrics included the rich-club coefficient, the degree, the global efficiency, the local efficiency, and the nodal efficiency. (G) For each participant, we obtained a $246 \times 246$ symmetric FC matrix with Pearson's correlation coefficients as the weights, and negative correlation coefficients were set as zero. (H) The alterations in SC-FC coupling in AD patients and $\mathrm{MCl}$ patients were investigated.

main diffusion direction from voxel to voxel in the brain mask. A streamline will terminate when the streamline reaches a voxel with an FA value $<0.1$, when the streamline exceeds the cerebral mask, or when the trajectory of the streamline makes a turn sharper than 45 degrees $(1,50)$.

\section{fMRI Preprocessing}

We used a toolbox for Data Processing \& Analysis for Brain Imaging (DPABI) to preprocess resting-state fMRI data following a method illustrated previously (51). The first 10 volumes were discarded due to the instability of the initial signals before starting the preprocessing. Then, slice timing correction and head motion correction were performed for each subject (52-55). According to the criteria of spatial movement, subjects with more than $3 \mathrm{~mm}$ or degrees of head movement in any direction were removed. Next, the T1 image was registered to the mean head motion-corrected fMRI image. The functional images were then resampled to 3-mm isotropic voxels and spatially smoothed with a 4-mm full-width half-maximum (FWHM) Gaussian kernel. We regressed several nuisance signals, such as global mean signals, head motion signals (Friston's 24-parameter model), and signals from the cerebrospinal fluid and white matter from the data. To reduce the effects of high-frequency noise and low-frequency drift, we used linear detrending and bandpass filtering (0.01$0.1 \mathrm{~Hz}$ ) (53). Finally, we extracted the mean time series from the 246 ROIs for each subject (56).

\section{Network Construction}

Structural connectivity (SC) was defined as at least 3 bundles of fibers $(>2)$ between any two regions (Figures 1A-E). In addition to analyzing unweighted networks, the weighted networks were calculated as the number of fibers divided by the sum of the surface areas of the 2 interconnected ROIs. Therefore, for each subject, we obtained a $246 \times 246$ weighted SC matrix.

Functional connectivity (FC) between any two nodes was computed as the Pearson correlation between the blood-oxygenlevel dependent (BOLD) time series (Figures 1C-G). For each participant, we obtained a $246 \times 246$ symmetric FC matrix with Pearson's correlation coefficients as the weights, and negative correlation coefficients were set as zero due to the ambiguous biological explanation $(57,58)$.

\section{Structural Network Analysis}

To investigate potential differences in the topology of the structural network between the patient groups and the $\mathrm{NC}$ group, characteristic graph metrics were calculated (binary and undirected $246 \times 246$ networks). We generated a binary and undirected network for each participant with the structural connectivity between two regions set as 1 if the corresponding weight was positive (weight $>0$ ). Graph metrics are introduced in detail elsewhere $(50,59)$. The investigated metrics included the rich-club coefficient (defined as the ratio of connections present between the remaining nodes and the total number of possible connections that would be present if the set was fully connected) (1), the degree (computed as the sum of the node's connections), the global efficiency (reflecting the ability for network-wide communication), the local efficiency (reflecting how much the network is fault tolerant and shows how efficient the communication is among the first neighbors of the node $i$ when it is removed), and the nodal efficiency (defined as the 
inverse of the harmonic mean of the minimum path length between an index node, $\mathrm{i}$, and all the other nodes in the network) (60). All graph metrics and null models were computed using the MATLAB-based GRETNA toolbox (http://www.nitrc.org/ projects/gretna/) (61). The results of the rich-club organization and nodal efficiency were visualized by the BrainNet Viewer toolbox (http://www.nitrc.org/projects/bnv) (62).

\section{Rich-Club Organization of Structural Network}

The "rich club" refers to nodes with higher degrees within brain networks and a higher connectivity strength of internodal connections compared to those composed of randomly selected brain regions (32). Rich-club nodes were chosen according to the top $18 \%$ of average node degree ranked nodes across all participants (26). According to the classification of richclub and non-rich-club nodes, structural network connections were categorized into three types of edges (32): (i) rich-club connections (red), connecting rich-club regions to each other; (ii) feeder connections (blue), connecting rich-club regions to non-rich-club regions; and (iii) local connections (green) connecting non-rich-club regions to each other (Figure 2D).

\section{SC-FC Relationship Analysis}

To measure the correlation between SC and FC and examine the alterations in SC-FC coupling in AD patients and MCI patients, we analyzed the correlation between the structural connection strength and functional connection strength of each network. This correlation was restricted by the connections with nonzero SC and nonzero FC. All nonzero entries of the SC matrix were selected, rescaled to a Gaussian distribution, and correlated with their nonzero functional counterparts selected from the FC matrix $(1,7,11,19-21,27,63-65)$. Therefore, for each of the subjects, we obtained a single SC-FC coupling metric.

\section{Statistical Analysis}

All statistical analyses in this study were performed by the Statistical Package for Social Science (SPSS, v20.0) (http://www. spss.com/). We used ANOVA to test for group differences in age and education and used a chi-square test to test for differences in sex. ANCOVAs (corrected for age, sex, and education) were used to test for group differences in richclub coefficients, normalized rich-club coefficients, three classes of connectivity density, other network topology metrics, nodal efficiency and SC-FC coupling, with Bonferroni corrections for multiple comparisons at $P<0.05$. We used partial Pearson's correlations controlling for sex, age, and education to measure how SC-FC coupling, graph metrics and clinical performance related to the density of connections in each group. Significance was set at $P<0.05$.

\section{RESULTS}

\section{Anatomical Rich-Club Organization}

The structural brain network construction process is illustrated in Figure 1D. Figures 2A,B show rich-club coefficient curves $R(k)$ and $R_{\text {norm }}(k)$ for NC (blue), MCI (yellow) and $\mathrm{AD}$ (red) groups.
In our results, anatomical rich-club organization was obvious in three groups, with the normalized rich-club coefficient $R_{\text {norm }}(k)$ increasing as a function of node degree $(k)$ higher than 1 . In the whole-brain network, the rich-club coefficient $R(k)$ showed a significant group difference $(k=5-8,10 ; P<0.05$, ANCOVA; age, sex and education as covariates; Bonferroni corrected). Significant group differences in normalized rich-club coefficients $R_{\text {norm }}(k)$ for the ranges $k=2,5-7,10$, and 14-16 reflect a higher level of connectivity between central hubs of the brain $(P<0.05$, ANCOVA; age, sex, and education as covariates; Bonferroni corrected). Therefore, the rich-club organization of the structural brain networks in patients was significantly altered. The results for 90 brain regions are shown in Supplementary Figures S1A,B.

\section{Rich-Club Regions in SC}

Rich-club regions were defined as the top $43(k>10)$ brain regions with the highest degree (Figure 2C, Table 2). Richclub nodes were distributed in the Subcortical Nuclei (18 nodes, including Hippocampus, Basal Ganglia, and Thalamus), Temporal Lobe (10 nodes were located in Fusiform Gyrus, Superior Temporal Gyrus and Middle Temporal Gyrus), Frontal Lobe (6 nodes, including Orbital Gyrus, Precentral Gyrus and Inferior Frontal Gyrus), Parietal Lobe (4 nodes, including Precuneus and Inferior Parietal Lobule), Occipital Lobe (4 nodes were concentrated in the Medioventral Occipital Cortex), and Insular Lobe (1 node). This result is similar to the results of previous studies using the same atlas (66). The results for 90 brain regions are shown in Supplementary Figure S1C.

\section{Density of Rich-Club, Feeder, and Local Connections}

Figure 2D shows the different classifications of structural connections in brain networks. In the three groups, richclub connections were found to include $11.32-11.72 \%$ of the total network density, the proportion of feeder connections ranged from 34.66 to $34.17 \%$, and the percentage of local connections ranged from 53.68 to $54.44 \%$ (Figure 2E). No significant differences were detected between the three groups.

Figure $2 \mathrm{~F}$ shows the mean (SD) density values for each of the 3 classes. The density of feeder $(F=5.435, P=0.006)$ and local $(F=4.199, P=0.018)$ connections revealed significant reductions (ANCOVA; age, sex, and education as covariates; Bonferroni corrected). No significant group differences were found in rich-club density $(F=1.941, P=0.149)$, and no significant reductions in rich-club density were found in the $\mathrm{AD}$ vs. MCI $(P=0.851)$ or NC groups $(P=0.065)$ and MCI vs. NC $(P=0.088)$ groups. For feeder and local density, there were significant decreases in the MCI (feeder: $P=0.036$; local: $P=0.014$ ) and AD (feeder: $P=0.002$; local: $P=0.021$ ) groups relative to the $\mathrm{NC}$ group. However, there was no significant difference in feeder $(P=0.143)$ and local $(P=0.749)$ density in the AD vs. MCI groups (ANCOVA; age, sex, and education as covariates; Bonferroni corrected). The results for 90 brain regions are shown in Supplementary Figures S1D-F. 
A

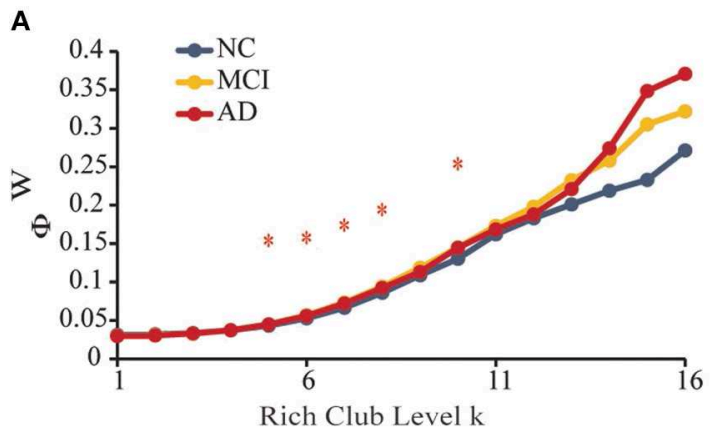

C

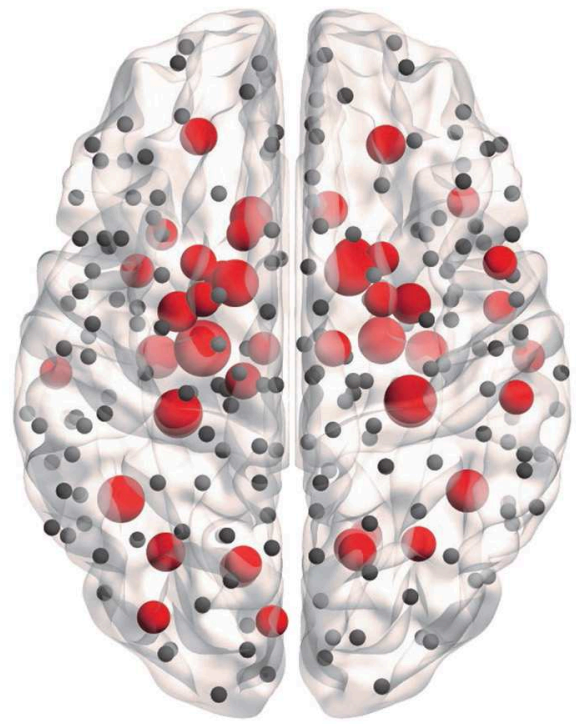

Rich club

Non-rich club

E

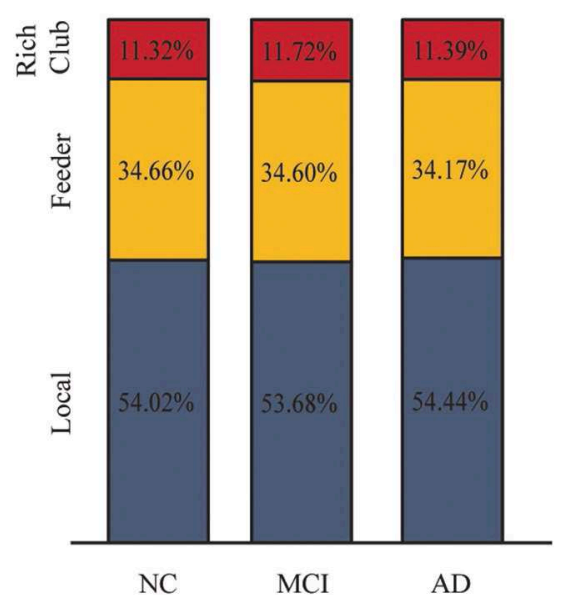

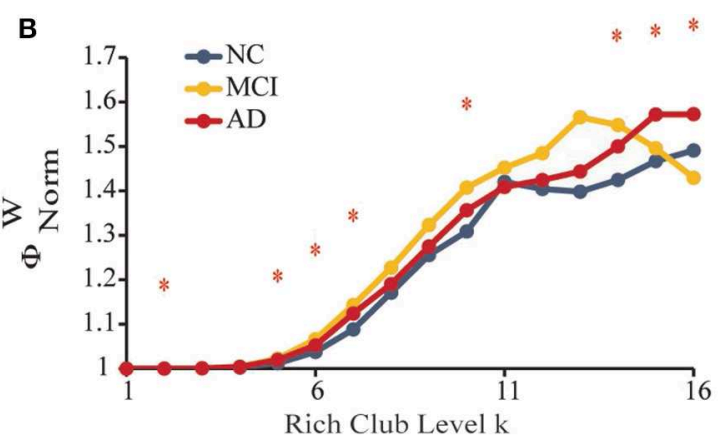

D

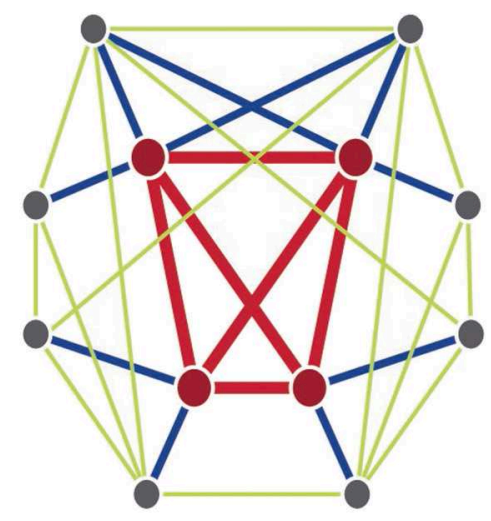

Rich club $\quad$ Feeder Local

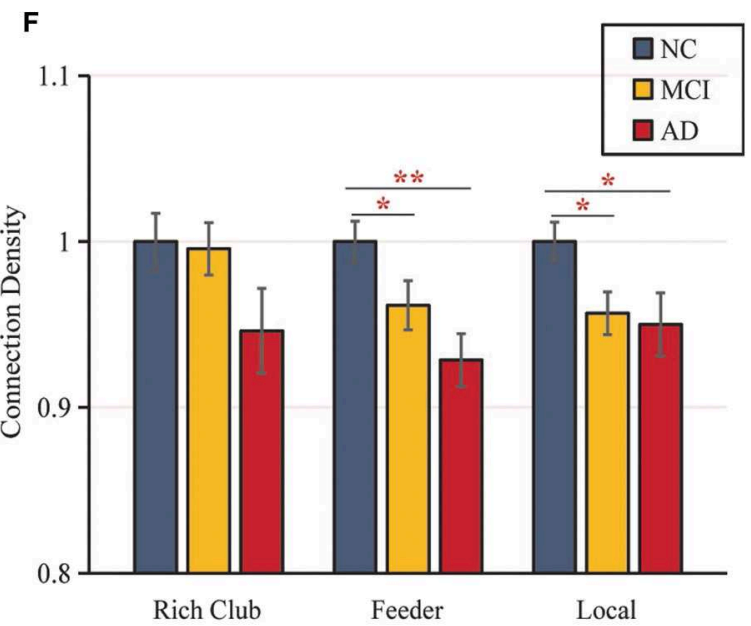

FIGURE 2 | Rich-Club Organization. (A) The rich-club coefficients. (B) The normalized rich-club coefficients. (C) The rich-club nodes (red nodes) are shown across all groups. (D) The different kinds of connections in structural networks. (E) The proportions of three kinds of connections for each group. (F) Bar graphs display the mean (SD) density of the rich-club, feeder and local connections $\left({ }^{\star \star} P<0.01,{ }^{\star} P<0.05\right)$. 
TABLE 2 | List of rich-club nodes.

\begin{tabular}{|c|c|c|c|c|}
\hline Abbreviations & Gyrus & Lobe & Average node degree & $\operatorname{MNI}(X, Y, Z)$ \\
\hline Hipp_L_2_1 & Hippocampus & Subcortical nuclei & 19.351 & $-22,-14,-19$ \\
\hline Hipp_R_2_2 & Hippocampus & Subcortical nuclei & 17.907 & $29,-27,-10$ \\
\hline Hipp_L_2_2 & Hippocampus & Subcortical nuclei & 16.464 & $-28,-30,-10$ \\
\hline Hipp_R_2_1 & Hippocampus & Subcortical nuclei & 15.948 & $22,-12,-20$ \\
\hline FuG_L_3_1 & Fusiform gyrus & Temporal lobe & 14.557 & $-33,-16,-32$ \\
\hline FuG_R_3_1 & Fusiform gyrus & Temporal lobe & 14.474 & $33,-15,-34$ \\
\hline OrG_L_6_5 & Orbital gyrus & Frontal lobe & 14.309 & $-10,18,-19$ \\
\hline BG_R_6_3 & Basal ganglia & Subcortical nuclei & 14.134 & $15,8,-9$ \\
\hline FuG_R_3_3 & Fusiform gyrus & Temporal lobe & 14.134 & $43,-49,-19$ \\
\hline FuG_L_3_2 & Fusiform gyrus & Temporal lobe & 12.639 & $-31,-64,-14$ \\
\hline MVOcC_L_5_5 & Medioventral occipital cortex & Occipital lobe & 12.619 & $-13,-68,12$ \\
\hline MVOcC_R_5_5 & Medioventral occipital cortex & Occipital lobe & 12.598 & $15,-63,12$ \\
\hline PCun_R_4_3 & Precuneus & Parietal lobe & 12.289 & $16,-64,25$ \\
\hline OrG_R_6_5 & Orbital gyrus & Frontal lobe & 12.206 & $9,20,-19$ \\
\hline OrG_L_6_3 & Orbital gyrus & Frontal lobe & 12.103 & $-23,38,-18$ \\
\hline PCun_L_4_3 & Precuneus & Parietal lobe & 11.948 & $-12,-67,25$ \\
\hline BG_L_6_3 & Basal ganglia & Subcortical nuclei & 11.835 & $-17,3,-9$ \\
\hline Tha_L_8_7 & Thalamus & Subcortical nuclei & 11.753 & $-12,-22,13$ \\
\hline Tha_R_8_7 & Thalamus & Subcortical nuclei & 11.68 & $10,-14,14$ \\
\hline BG_L_6_4 & Basal ganglia & Subcortical nuclei & 11.619 & $-23,7,-4$ \\
\hline BG_L_6_1 & Basal ganglia & Subcortical nuclei & 10.577 & $-12,14,0$ \\
\hline STG_R_6_3 & Superior temporal gyrus & Temporal lobe & 10.577 & $51,-4,-1$ \\
\hline IPL_L_6_1 & Inferior parietal lobule & Parietal lobe & 10.392 & $-34,-80,29$ \\
\hline Tha_L_8_4 & Thalamus & Subcortical nuclei & 10.361 & $-7,-14,7$ \\
\hline MTG_R_4_4 & Middle temporal gyrus & Temporal lobe & 10.351 & $58,-16,-10$ \\
\hline MTG_L_4_4 & Middle temporal gyrus & Temporal lobe & 10.34 & $-58,-20,-9$ \\
\hline MVOcC_R_5_4 & Medioventral occipital cortex & Occipital lobe & 10.32 & $18,-60,-7$ \\
\hline IFG_R_6_5 & Inferior frontal gyrus & Frontal lobe & 10.278 & $42,22,3$ \\
\hline STG_L_6_1 & Superior temporal gyrus & Temporal lobe & 10.144 & $-32,14,-34$ \\
\hline MVOcC_L_5_2 & Medioventral occipital cortex & Occipital lobe & 10.034 & $-5,-81,10$ \\
\hline
\end{tabular}

\section{Structural Network Graph Metrics}

Disrupted topological organization of brain networks leads to altered information transmission efficiency. Group differences (ANCOVA; age, sex, and education as covariates; Bonferroni corrected) were observed in the global efficiency ( $F=3.301$, $P=0.041)$, local efficiency $(F=5.986, P=0.004)$ and degree $(F=5.484, P=0.006)$. Significant reductions in global efficiency (MCI vs. NC: $P=0.023 ; \mathrm{AD}$ vs. NC: $P=0.050$ ), local efficiency (MCI vs. NC: $P=0.027$; AD vs. NC: $P=0.001$ ) and degree (MCI vs. NC: $P=0.017$; AD vs. NC: $P=0.003$ ) were observed in the two patient groups compared to the NC group (ANCOVA; age, sex, and education as covariates). However, there was no significant difference between $\mathrm{MCI}$ and $\mathrm{AD}$ in these three topological properties (global efficiency: $P=0.904$; local efficiency: $P=0.13$; degree: $P=0.275$ ) (Figure 3A). The results for 90 brain regions are shown in Supplementary Figure S2A. 
A
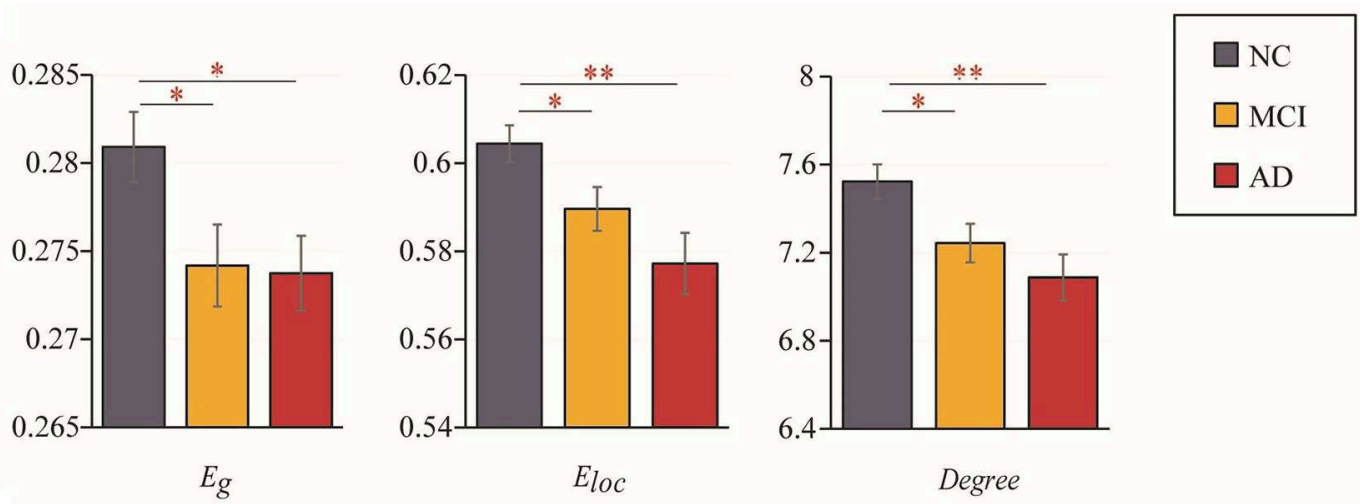

B
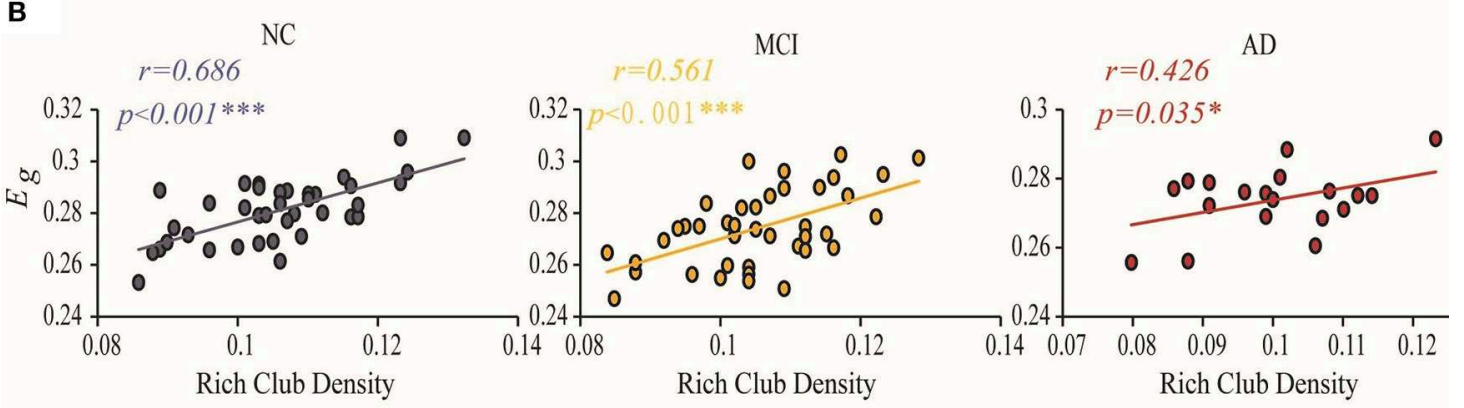

FIGURE 3 | Efficiency and rich-club density. (A) Bar graphs display the mean (SD) global efficiency, local efficiency and degree. (B) The correlations between the global efficiency and the density of rich-club connections. The star-labeled numbers represent significant correlations $\left({ }^{\star} P<0.05,{ }^{* \star} P<0.01,{ }^{* \star *} P<0.001\right)$.

\section{Relationship Between Global Efficiency and Rich-Club Density}

We further investigated the relationship between the changes in rich-club organization and topologic alterations of the brain structural connectome. Figure 3B shows that the correlation of global efficiency to rich-club density decreased with worsening of disease status. A significantly positive correlation (with age, sex and education as covariates) was found between global efficiency and the rich-club density in the NCs $(r=0.686, P<0.001)$, MCI patients $(r=0.561, P<0.001)$ and AD patients $(r=0.426$, $P=0.035)$. The results for 90 brain regions are shown in Supplementary Figure S2B.

\section{Altered SC-FC Coupling and Relationship to the Feeder and Local Density}

Group differences (ANCOVA; age, sex, and education as covariates; Bonferroni corrected) were observed for the SC-FC coupling of all connections $(F=13.164, P<0.001)$, feeder connections $(F=3.521, P=0.034)$ and local connections $(F=8.325, P<0.001)$. But the SC-FC coupling of rich-club connections $(F=1.863, P=0.161)$ did not have group difference.

Under the constraint of existing structural connections, significant increases in the strength of SC-FC coupling were found (MCI vs. NC: $P=0.033$; AD vs. NC: $P<0.001$; $\mathrm{AD}$ vs. MCI: $P=0.011)$. Furthermore, the significantly increased SC-FC coupling was concentrated in feeder connections (AD vs. NC:
$P=0.023 ; \mathrm{AD}$ vs. MCI: $P=0.013)$ and local connections (AD vs. NC: $P<0.001$; MCI vs. NC: $P=0.011$ ) (Figure 4A).

The correlation (with age, sex, and education as covariates) of SC-FC coupling to feeder density (NC: $r=0.559, P<0.001$; MCI: $r=0.311, P=0.025$; AD: $r=0.023, P=0.463)$ and local density (NC: $r=0.581, P<0.001$; MCI: $r=0.188, P=0.122$; AD: $r=-0.088, P=0.360$ ) were decreased (Figure 4B). The results for 90 brain regions are shown in Supplementary Figure S3.

\section{Alzheimer's Disease-Related Alterations in Nodal Efficiency of SC}

We further examined the structural brain network nodes showing significant differences in nodal efficiency (Figure 5), following the discovery of impaired rich-club organization. Group differences (ANCOVA; age, sex, and education as covariates; Bonferroni corrected) were observed for the 44 abnormal nodes, including 5 rich-club regions, IFG_R_6_5, the bilateral FuG_3_1, FuG_L_3_2, and INS_L_6_6, and 39 nonrich-club regions, which were located in the Temporal Lobe (10 nodes), Frontal Lobe (8 nodes), Insular Lobe ( 8 nodes), Limbic Lobe (5 nodes), Parietal Lobe (4 nodes), Occipital Lobe (2 nodes) and Subcortical Nuclei (1 node) (Figure 5A).

In the MCI group, 18 abnormal regions were found compared to the NC group, including 2 rich-club regions, IFG_R_6_5 and FuG_R_3_1, and 16 non-rich-club regions (Figure 5B). We found that the AD group had more abnormal nodes than the MCI group. When the AD patients were compared to the 

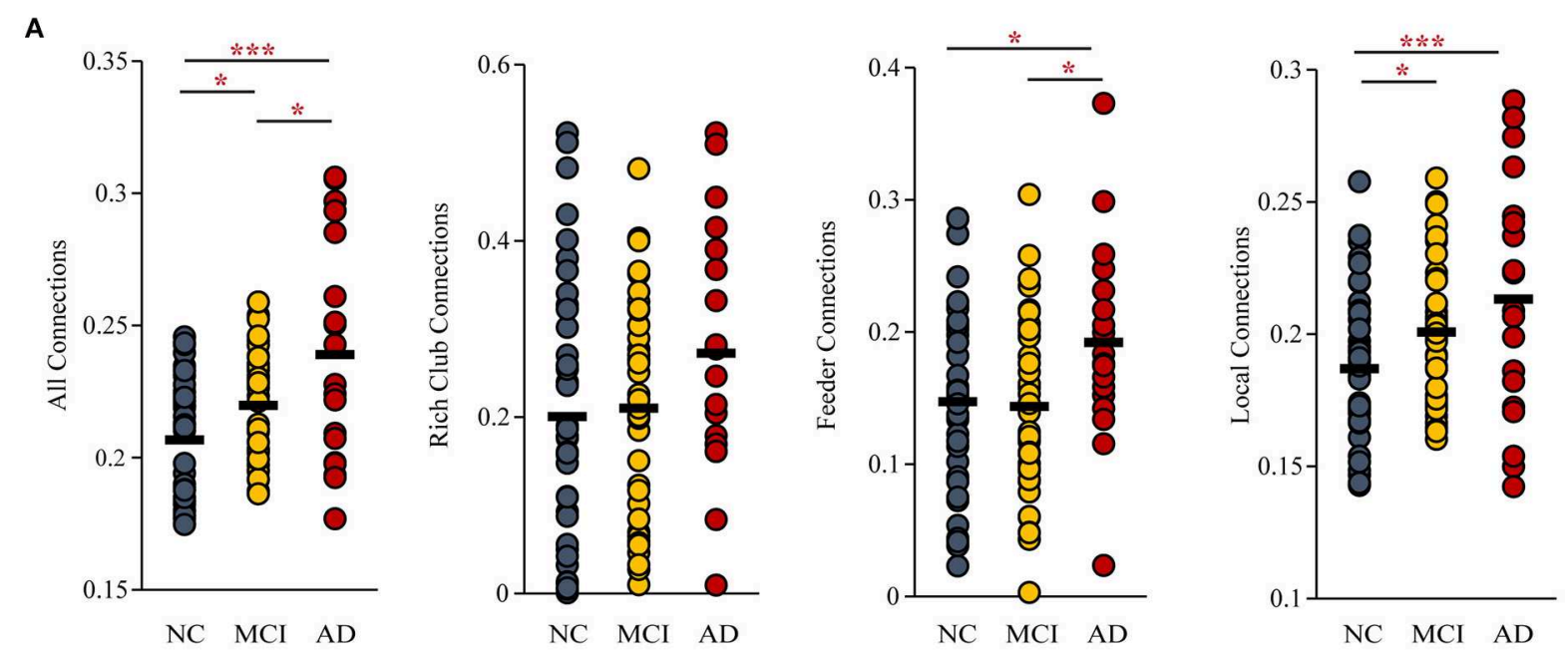

B
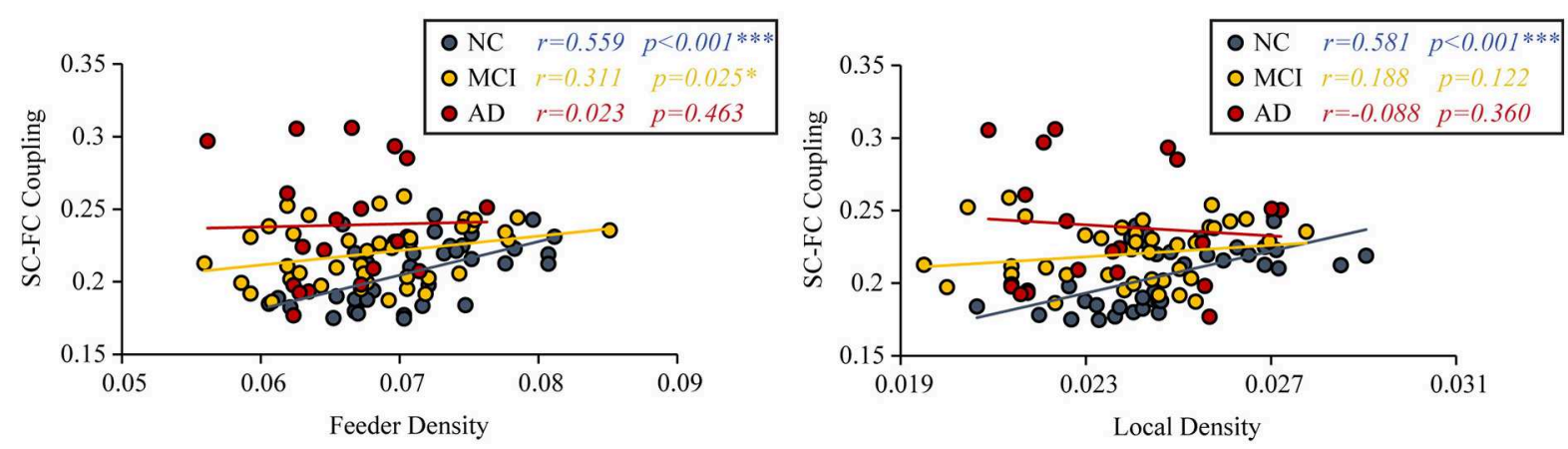

FIGURE 4 | SC-FC Coupling. (A) MCl and AD patients had significantly increased SC-FC coupling compared with the NC group. (B) The correlations between the SC-FC coupling and the feeder and local density. The star-labeled numbers represent significant correlations $\left({ }^{\star} P<0.05,{ }^{\star \star} P<0.01,{ }^{\star \star \star} P<0.001\right)$.

NCs, the 8 rich-club regions with altered nodal efficiency were Hipp_R_2_1, IFG_R_6_5, STG_R_6_3, MTG_R_4_4, bilateral FuG_3_1, FuG_L_3_2, and INS_L_6_6. The 38 non-rich-club regions were concentrated in the Temporal Lobe (14 nodes), Frontal Lobe (6 nodes), Parietal Lobe (5 nodes), Insular Lobe (8 nodes), Limbic Lobe (3 nodes), Subcortical Nuclei (1 node), and Occipital Lobe (1 node) (Figure 5C). Only 5 abnormal nonrich-club regions were found in $\mathrm{AD}$ patients compared with MCI patients (Figure 5D). The results for 90 brain regions are shown in Supplementary Figure S4.

\section{Relationship Among Connection Density, Coupling Metrics, and Clinical Performance}

To investigate the relationship of the clinical and cognitive test variables and the altered brain networks in patients, we correlated the clinical and cognitive test variables with the anatomical rich-club organization and coupling strength (with age, sex, and education as covariates) (Figure 6, Table 3). In patients, the rich-club connection density was significantly correlated with FAQ and MMSE scores, the feeder connection density was significantly correlated with the CDR, FAQ, MMSE, and MoCA scores, and the local connection density was significantly correlated with the CDR, FAQ, and MMSE scores (Figure 6A). The coupling strength of all connections, feeder connections and local connections were significantly correlated with the CDR, FAQ, MMSE, and MoCA scores (Figure 6B). However, no significant correlations were found between the coupling strength of rich-club connections and any clinical variables. The results for 90 brain regions are shown in Supplementary Figure S5, Supplementary Table S1.

\section{DISCUSSION}

We investigated patterns of $\mathrm{AD}$-related changes in brain structural networks and functional brain dynamics. Our main findings are as follows: (1) The feeder and local density were significantly decreased. Disrupted topological organization of structural networks leads to low information transmission efficiency. (2) Compared with NCs, MCI, and AD patients 


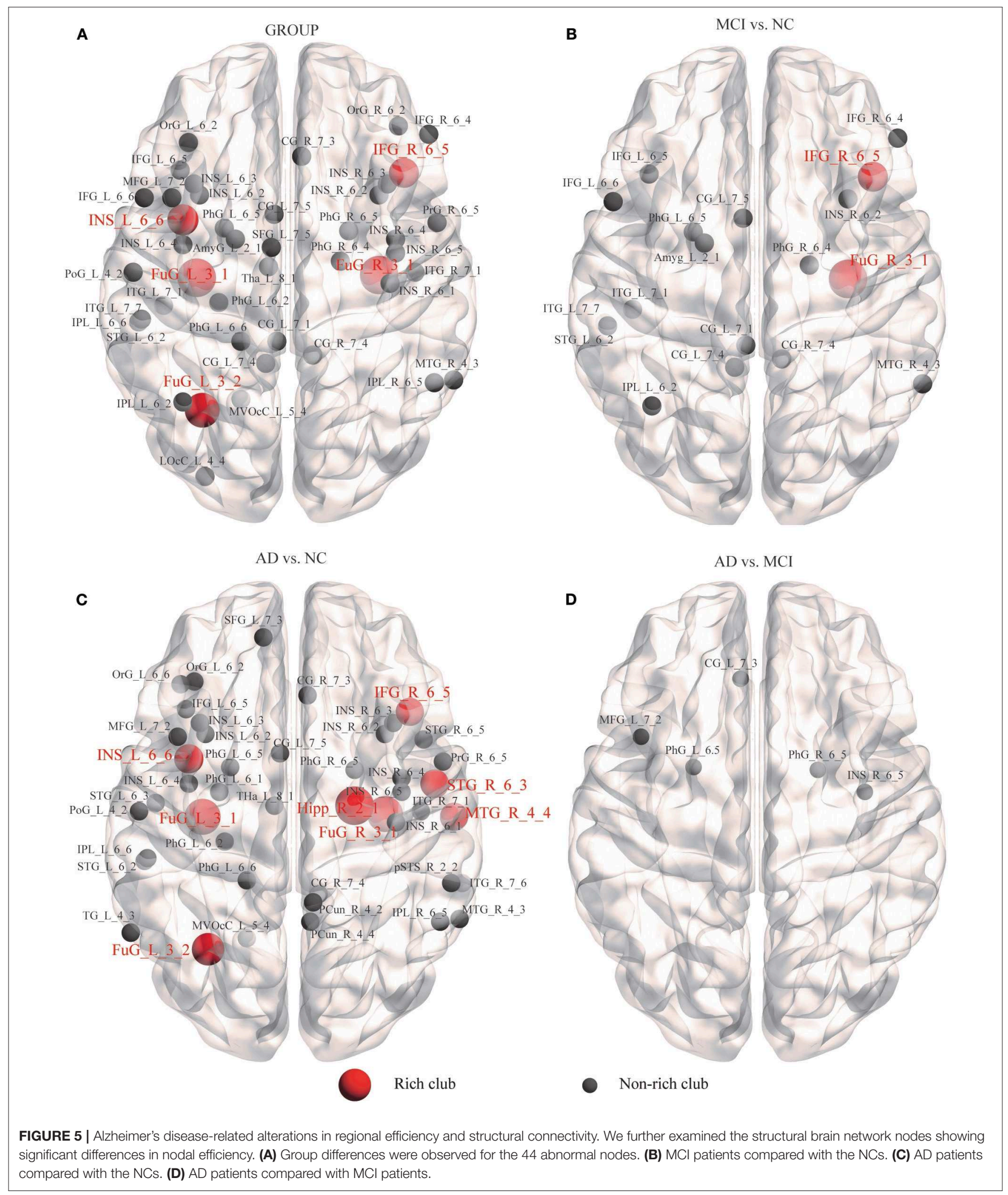

exhibited an increase in SC-FC coupling that was concentrated on the feeder and local connections. SC-FC coupling also displayed significant differences between $\mathrm{MCI}$ and $\mathrm{AD}$ patients that were mainly focused on abnormal feeder connections. (3) The alterations of regional efficiency in patients were observed, and most were peripheral regions. (4) The connection density 
A

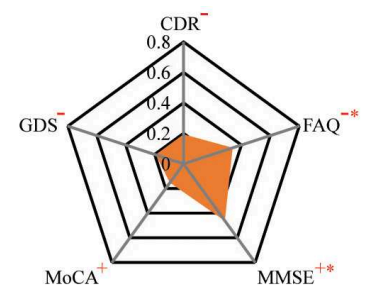

Rich Club Density

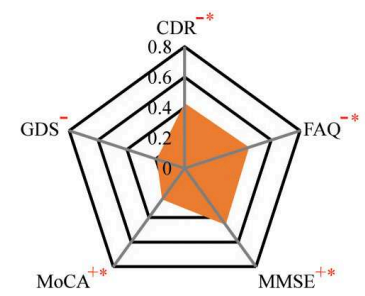

Feeder Density

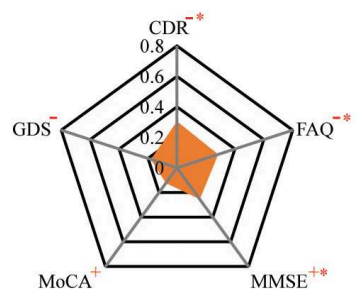

Local Density

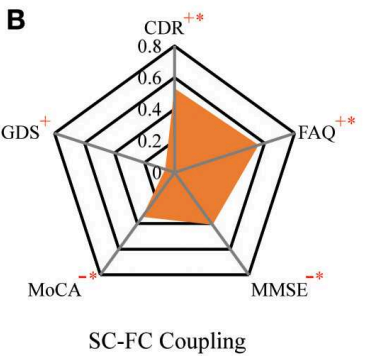

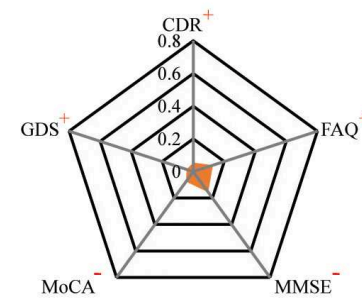

Rich Club Coupling

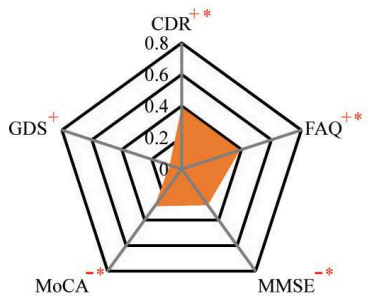

Feeder Coupling

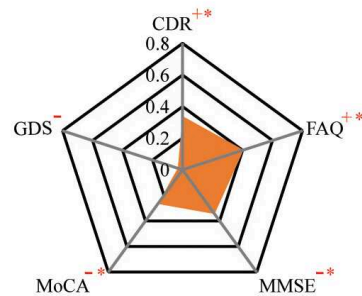

Local Coupling

FIGURE 6 | The relationship between connection density and coupling metrics and clinical performance. (A) The correlation between the clinical performance and the connection density. (B) The correlation between the clinical performance and the coupling metrics.

TABLE 3 | Partial Pearson's correlations between connection density and coupling metric with clinical performance.

\begin{tabular}{|c|c|c|c|c|c|c|}
\hline COV: Sex \& Age & & CDR & FAQ & MMSE & MoCA & GDS \\
\hline \multirow[t]{2}{*}{ Rich-club density } & $r$ & 0.193 & 0.339 & 0.461 & 0.152 & 0.192 \\
\hline & $P$ & 0.099 & $0.011^{*}$ & $0.001^{* *}$ & 0.157 & 0.101 \\
\hline \multirow[t]{2}{*}{ Feeder density } & $r$ & 0.428 & 0.446 & 0.459 & 0.252 & 0.193 \\
\hline & $P$ & $0.001^{\star *}$ & $0.001^{\star \star}$ & $0.001^{* *}$ & $0.046^{\star}$ & 0.100 \\
\hline \multirow[t]{2}{*}{ Local density } & $r$ & 0.304 & 0.284 & 0.254 & 0.123 & 0.179 \\
\hline & $P$ & 0.020 * & $0.028^{*}$ & $0.044^{*}$ & 0.208 & 0.117 \\
\hline \multirow[t]{2}{*}{ SC-FC coupling } & $r$ & 0.531 & 0.559 & 0.409 & 0.347 & 0.062 \\
\hline & $P$ & $<0.001^{\star \star \star *}$ & $<0.001^{\star \star *}$ & $0.002^{\star \star}$ & $0.009^{\star *}$ & 0.342 \\
\hline \multirow[t]{2}{*}{ Rich-club coupling } & $r$ & 0.054 & 0.126 & 0.155 & 0.075 & 0.042 \\
\hline & $P$ & 0.361 & 0.201 & 0.153 & 0.310 & 0.391 \\
\hline \multirow[t]{2}{*}{ Feeder coupling } & $r$ & 0.392 & 0.389 & 0.283 & 0.291 & 0.079 \\
\hline & $P$ & $0.004^{\star \star}$ & $0.004^{\star \star}$ & $0.028^{\star}$ & $0.025^{\star}$ & 0.302 \\
\hline \multirow[t]{2}{*}{ Local coupling } & $r$ & 0.339 & 0.406 & 0.345 & 0.266 & 0.031 \\
\hline & $P$ & $0.011^{*}$ & $0.003^{\star \star}$ & $0.009^{* *}$ & $0.037^{*}$ & 0.420 \\
\hline
\end{tabular}

Partial Pearson's correlations controlled for age and sex were used to assess how connection density and coupling related to clinical performance. The star-labeled numbers represent significant correlations $\left({ }^{\star} P<0.05,{ }^{\star \star} P<0.01\right.$, $\left.{ }^{\star \star \star} P<0.001\right)$. Bold Font indicated significant correlation $(P<0.05)$.

and coupling strength were significantly correlated with clinical metrics in patients.

\section{Altered Anatomical Rich-Club Organization}

An increasing normalized rich-club coefficient $\left(R_{\text {norm }}\right)>1$ over a range of degrees $(\mathrm{k})$ reflects the existence of a rich-club organization in structural brain networks $(1,36,50)$. Compared with NCs, the rich-club organization of the structural brain networks in patients was significantly altered, as indicated by the striking group difference in rich-club coefficients. To further confirm our findings, we selected 43 nodes with an average node degree $>10$ in the structural network across all participants as the hub node. Rich-club nodes mainly included the Hippocampus, Precuneus, and Fusiform Gyrus, which were consistent with previous studies $(26,67)$. In addition to the rich-club connection, the feeder and local connections showed more significantly damaged in MCI and AD patients (Figure 2F), which was consisted with previous studies. (26) found that the brain regions with the most aberrant connections involving peripheral and rich club regions in patients compared with NC were distributed throughout the whole brain. Moreover, most significant differences in nodal efficiency were observed in peripheral regions. $\mathrm{AD}$ is considered as a disease of extensive connectivity disorders, that peripheral regions and connections were the most vulnerable even in preclinical stages, the peripheral regions are more likely to suffer disruptions.

The node degree of MCI and AD patients was significantly reduced, indicating that the topological organization of the patient was damaged. Disrupted topological organization of brain networks affects the integration of information propagated among distant brain regions $(68,69)$. After the brain's longdistance signal traffic passed through a focal network, particularly in the central rich club, it depended on the feeder connections to facilitate or promote a variant of neuronal information for different parts of the network (32). We further investigated the relationship between the changes in anatomical rich-club organization and topologic alterations of the brain structural connectome and found that the brains of patients seemed to be less efficiently organized. The efficiency and degree of the MCI and AD patients were significantly decreased compared to those of the NC patients, and this finding was consistent with previous graph theory studies on $\operatorname{AD}(29,30)$. We found that 
the decrease in structural network global efficiency was closely associated with the decrease in three types of connection density. As shown in Figure 2E, the proportion of the three connections in the three diagnostic groups remained generally stable. There were slight differences (but not significantly differences) of the rich-club density in the $\mathrm{MCI}$ and $\mathrm{AD}$ patients decreased relative to the NCs, so not all connections contributing to the highly interconnected nodes of the rich-club were kept intact during the stages of the disease. The failure of a rich-club region can have a severe effect on the level of global communication efficiency of a network due to its central role in the network $(70,71)$. More importantly, we found that with worsening of disease status from $\mathrm{MCI}$ to $\mathrm{AD}$, the correlation between the global efficiency and the rich-club density was reduced. Corresponding to previous studies, the more central regions of the rich-club, which connect to remote nodes, may be relatively resistant to the neurodegenerative process (36). Peripheral regions were more susceptible due to their reduced persistence and lower level in the hierarchical network (26). Therefore, the rich-club connections are more stable than the feeder and local connections. As the condition worsened, the damage to the feeder and local connections intensified, and the impact on the global efficiency far exceeded the impact of the abnormal rich-club connections.

These findings indicated that the main cause of the lower global efficiency of the patient was the abnormal disruption of the overall structural connectivity. However, the rich-club density did not decrease significantly in the patients, suggesting that the most important factors were the densities of the feeder and local connections.

\section{The Relationship Between the Increase in SC-FC Coupling and Rich-Club Organization}

We observed an increased level of coupling between SC and FC in the MCI and AD patients, which was consistent with previous studies $(21,27)$. Moreover, Dai et al. reported that the increased SC-FC coupling of the default-mode network in AD patients indicated that $\mathrm{AD}$ leads to a strengthened relationship between FC and the underlying anatomical connectivity $(21,27)$. However, one previous study reported the opposite result: a decrease in the strength of SC-FC coupling in AD patients (11). We found that the method of network construction in this study was different from the other two studies, and we speculated that these differences might lead to inconsistent findings. The increased coupling means a loss of reorganization of the brain network in AD and MCI (72), which likely reflects a worsening of disease status. Specifically, the degree of SC integrity might reflect the capacity of the cerebral cortex to maintain functional organization diversity or neural activity interactions $(27,73)$. Therefore, the increased coupling may suggest that the disease leads to functional interactions that are more directly related to the underlying anatomical connectivity of the brain and may be indicative of more stringent and less dynamic brain function and less functional network reorganization in patients $(1,21)$.

More importantly, further analysis revealed that the increased SC-FC coupling in the MCI and AD patients was concentrated on the feeder and local connections, which indicated that SC-FC coupling may detect subtle brain connectivity disruption with greater sensitivity than does a single modality $(21,27)$. Specifically, the change in the correlation between the structural network and the functional network of the NCs and MCI patients was mainly focused on abnormal local connections. The feeder connections of the structural network of MCI patients were also damaged, but there was no significant change in the SC-FC coupling of the feeder connections. We speculate that this observation was due to the compensation mechanism of the brain; patients also present adaptive network reorganization associated with relatively preserved cognitive function (74). However, these mechanisms are transient and attenuated (72). Compared with $\mathrm{MCI}$, feeder connections are the main reason for the increase in SC-FC coupling in $\mathrm{AD}$ patients. As disease status worsens from MCI to $\mathrm{AD}$, the disturbance of the feeder and local connections of the structural network become more serious, and the corresponding functional connections are greatly affected $(7,75)$. Therefore, the increase in SC-FC coupling in AD patients was mainly concentrated on feeder and local connections compared to that in NCs. Our study of changes in SC-FC coupling may contribute to a more accurate understanding of the dynamic changes in brain network structure in patients.

With worsening of disease status from MCI to AD, the coupling strengths were decreasingly correlated with density in the feeder and local connection, indicating a lower density of feeder and local connections accompanied by altered functional brain dynamics. Consistent with previous studies, destruction of feeder and local connections would contribute to the disruption of an optimal structural organization in $\mathrm{AD}$ and MCI patients (36). That an increased level of SC-FC coupling may be related to the destruction of the optimal structure led to not only weaker neural communication (27) but also lower functional brain dynamics. The significant correlation between the feeder connections and the SC-FC coupling disappeared in the $\mathrm{AD}$ patients, and the significant correlation between the local connections and the SC-FC coupling disappeared during the MCI phase. These results confirmed the previous speculation that MCI patients' structure-feeder connection still maintains relatively stable brain functional dynamics due to the compensation mechanism of the brain. Alternatively, FC is more robust and resilient against pathological attacks than SC (76), and FC might be less vulnerable and may even serve as a compensation mechanism for reduced SC in the face of early cognitive decline (77).

\section{Alterations of Regional Efficiency of SC in Patients}

Through the analysis of nodal efficiency, significant group differences were observed for a large number of abnormal nodes, and most were peripheral regions, suggesting that $\mathrm{AD}$ is a disease of widespread connectivity disorders mainly based on feeder and local connections, which corresponded to our previous results. The efficiency of nodes such as the inferior frontal gyrus, amygdala, and temporal lobe (parahippocampal gyrus, superior temporal gyrus, parahippocampal gyrus) in the MCI patients was abnormally altered, which reflected the 
abnormality of the connections in these regions. These regions are involved in high-level cognitive functions, such as episodic memory, attention, motivation, self-awareness, and audiovisual integration, which are the main deficiencies in patients (e.g., frontal lobe, temporal lobe and insula). Previous studies have demonstrated $\mathrm{AD}$-related abnormalities in the frontal lobe (35, $78)$, temporal lobe $(79-81)$, and insula $(35,82)$. Moreover, with worsening of disease status from MCI to $\mathrm{AD}$, the involvement of more nodes, and more severe damage to the structural brain network, the $\mathrm{AD}$ patients have more abnormal nodes than the MCI patients, including the precuneus, hippocampus and middle temporal gyrus, which are related to memory and cognitive function. The precuneus is involved in not only the rich-club nodes of the brain organization in this study but also the central nodes of the default-mode network in previous studies, and these regions have been implicated in high-level cognitive functions, including episodic memory, self-related processing, and aspects of consciousness (83). The frontal regions (e.g., middle frontal gyrus (MFG)) are thought to be involved in emotion, memory, and executive functions $(84,85)$. Many previous studies have demonstrated that these frontal regions exhibited AD-related abnormalities in structural network integrity $(86,87)$, gray matter morphology (88), and functional interactions (89-91).

\section{Association of Structural Connection Density With Coupling and Clinical Metrics} Abnormal connection densities were correlated with all the clinical and cognitive test variables in patients. The high correlations between the structural connection densities and the behavioral scores indicated that the connection densities were highly associated with the disrupted cognitive/memory functions (78). Consistent with previous studies, these findings converged on the notion that all $\mathrm{AD}$-related patients present widespread aberrant connections involving the peripheral regions, which may contribute to the early decline in memory that they experience (26). The number of clinical scales significantly associated with feeder and local density was more than that associated with rich-club density, which was consistent with our findings. We hypothesized that feeder and local connections are more susceptible to damage in the course of the disease and thus have a greater impact on cognitive performance, while rich-club connections are more robust. The stable rich-club connections help to maintain the core organization of the brain when other rich-club regions become disrupted (50). Human SC is closely related to FC (92) and underlies high-order cognitive activities (93). Therefore, our results of disrupted SC may reflect the interruption of functional connections that affects the cognitive performance of the patients. The rich-club regions of the brain play a core role in optimizing global brain communication and are associated with higher cognitive functions (21). Abnormal rich-club region can have a serious effect on the brain's higher cognitive function due to its central role in the network. Although there was no significant difference in the rich-club density in our study, there was still a downward trend. Therefore, we found that rich-club density was significantly correlated with the
FAQ and MMSE scores, and these results did not contradict our conclusions.

To our knowledge, this is the first study demonstrating a close association between greater SC-FC coupling and neuropsychological tests, including the CDR, FAQ, MMSE, and MoCA in patients. The results indicated that with the development of the disease, the level of the correlation between the structure and function of the brain increases, brain function becomes more stringent and less dynamic, and less functional network reorganization occurs in patients. Moreover, the clinical manifestations of patients were more obvious. Similarly, we observed that the coupling strength of the feeder and local connections were also significantly correlated with the four neuropsychological tests. However, the SC-FC coupling of the rich-club connections was not significantly correlated with any scale score. This finding is consistent with our results and further demonstrates that our results are reasonable and credible. SC-FC coupling may provide a potential biomarker that is more sensitive than a single modality to uncover the pathophysiology of $\mathrm{AD}$ $(1,19)$.

\section{Limitations and Future Work}

Although we provided information on additional network properties and functional network reorganization in patients with $\mathrm{MCI}$ and $\mathrm{AD}$, there were some limitations in the present study. The number of $\mathrm{AD}$ patients is quite small compared to the number of MCI patients and healthy controls. Regarding the limitation of the dataset, we are unable to collect more suitable $\mathrm{AD}$ data for experimentation. Whether the organization of SC and FC in patients have selective disruptions that disproportionately involve module-related or node-related connections remains to be determined. In our study, we calculated the correlation between SC and FC as SC-FC coupling, which was limited by the connections with non-zero SC and FC. As reported by Honey et al. (7) strong functional connections commonly exist between regions with no direct structural connection. Furthermore, due to the limitations of the method, there is currently no way to calculate the relationship between the functional connections and the indirect structural connections. The goal of these studies was simply to focus on the relationship between functional connections and structural connections. We will add the case of the indirect connections to the calculation of the SC-FC coupling in the next study. Besides, we have not studied whether the use of higher thresholds for functional connections will affect the results of SC-FC coupling. We plan to conduct such research in the future to improve the robustness of the results. Moreover, due to the crosssectional design we used, the causal relationship between the abnormal rich-club organization and accompanying changes in brain dynamics remains unclear.

\section{CONCLUSION}

In conclusion, our study found decreased levels of feeder and local connectivity in MCI and $\mathrm{AD}$ patients, which were the main contributing factors to the lower efficiency of the brain structural network. Disrupted topological organization of brain 
networks leads to low information transmission efficiency. The alterations of regional efficiency in patients further confirmed our findings. The MCI patients showed an intermediate position between the NC group and $\mathrm{AD}$ patients, and the $\mathrm{AD}$ patients had more abnormal nodes, including the rich-club nodes, than the MCI patients. Another important finding was that we have more accurately characterized the changing pattern of functional brain dynamics. The enhanced coupling between SC and FC in MCI and $\mathrm{AD}$ patients might be due to disruptions in optimal structural organization. More interestingly, we also found increases in the SC-FC coupling of feeder local connections in MCI and AD patients. SC-FC coupling also displayed significant differences between $\mathrm{MCI}$ and $\mathrm{AD}$ patients that were mainly focused on abnormal feeder connections. These findings suggest that robust rich-club and feeder connections help maintain stable brain functional dynamics. Finally, we examined whether the connection density and coupling strength were significantly correlated with clinical metrics in patients. In summary, our findings suggest that $\mathrm{AD}$ may break the hierarchical structure of the brain network, leading to functional network reorganization and communication network abnormally. SC-FC coupling may provide a potential biomarker that is more sensitive than a single modality to discover the pathophysiology of $\mathrm{AD}$. The present findings enhanced our understanding of the neurophysiologic mechanisms associated with $\mathrm{MCI}$ and $\mathrm{AD}$ from a brain network perspective.

\section{DATA AVAILABILITY STATEMENT}

Publicly available datasets were analyzed in this study. This data can be found here: http://adni.loni.usc.edu/.

\section{ETHICS STATEMENT}

The studies involving human participants were reviewed and approved by The Alzheimer's Disease Neuroimaging Initiative (ADNI) database. The patients/participants provided their written informed consent to participate in this study.

\section{AUTHOR CONTRIBUTIONS}

BW, RC, JX, and XW designed the study. RC, XW, YG, and JX did the experiments and data acquisition. XW, YG, and HZ performed analysis and interpretation of data. XW, YG, TL, and $\mathrm{WH}$ wrote the draft of the manuscript. TL, YX, and JW

\section{REFERENCES}

1. Mp VDH, Sporns O, Collin G, Scheewe T, Mandl RC, Cahn W, et al. Abnormal rich club organization and functional brain dynamics in schizophrenia. JAMA Psychiat. (2013) 70:783-92. doi: 10.1001/jamapsychiatry.2013.1328

2. Sporns O, Tononi G, Kötter R. The human connectome: a structural description of the human brain. PLoS Comput Biol. (2005) 1:e42. doi: 10.1371/journal.pcbi.0010042 contributed to revise. All authors approve the manuscript to be published and agreed on all aspects of the work.

\section{FUNDING}

This research was supported by the National Natural Science Foundation of China (61503272, 61672374, 61873178, and 61876124), the Natural Science Foundation of Shanxi (201801D121135 and 201901D111093), the International Science and Technology Cooperation Project of Shanxi (201803D421047), and the Youth Science and Technology Research Fund (201701D221119). Data collection and sharing for this project were funded by the Alzheimer's Disease Neuroimaging Initiative (ADNI; National Institutes of Health Grant U01 AG024904) and DOD ADNI (Department of Defense award number W81XWH-12-2-0012). ADNI was funded by the National Institute on Aging and the National Institute of Biomedical Imaging and Bioengineering and through generous contributions from the following: AbbVie, Alzheimer's Association; Alzheimer's Drug Discovery Foundation; Araclon Biotech; BioClinica, Inc.; Biogen; BristolMyers Squibb Company; CereSpir, Inc.; Cogstate; Eisai Inc.; Elan Pharmaceuticals, Inc.; Eli Lilly and Company; EuroImmun; F. Hoffmann-La Roche Ltd. and its affiliated company Genentech, Inc.; Fujirebio; GE Healthcare; IXICO Ltd.; Janssen Alzheimer Immunotherapy Research and Development, LLC.; Johnson \& Johnson Pharmaceutical Research and Development LLC.; Lumosity; Lundbeck; Merck \& Co., Inc.; Meso Scale Diagnostics, LLC.; NeuroRx Research; Neurotrack Technologies; Novartis Pharmaceuticals Corporation; Pfizer Inc.; Piramal Imaging; Servier; Takeda Pharmaceutical Company; and Transition Therapeutics. The Canadian Institutes of Health Research provides funds to support ADNI clinical sites in Canada. Private sector contributions are facilitated by the Foundation for the National Institutes of Health (www.fnih.org). The grantee organization is the Northern California Institute for Research and Education, and the study is coordinated by the Alzheimer's Therapeutic Research Institute at the University of Southern California. ADNI data are disseminated by the Laboratory for Neuro Imaging at the University of Southern California.

\section{SUPPLEMENTARY MATERIAL}

The Supplementary Material for this article can be found online at: https://www.frontiersin.org/articles/10.3389/fneur. 2020.00053/full\#supplementary-material 
6. Van Dijk KR, Hedden T, Venkataraman A, Evans KC, Lazar SW, Buckner RL. Intrinsic functional connectivity as a tool for human connectomics: theory, properties, and optimization. J Neurophysiol. (2010) 103:297-321. doi: 10.1152/jn.00783.2009

7. Honey CJ, Sporns O, Cammoun L, Gigandet X, Thiran JP, Meuli $\mathrm{R}$, et al. Predicting human resting-state functional connectivity from structural connectivity. Proc Natl Acad Sci USA. (2009) 106:2035-40. doi: 10.1073/pnas.0811168106

8. Hagmann P, Sporns O, Madan N, Cammoun L, Pienaar R, Wedeen VJ, et al. White matter maturation reshapes structural connectivity in the late developing human brain. Proc Natl Acad Sci USA. (2010) 107:19067-72. doi: 10.1073/pnas.1009073107

9. Honey CJ, Thivierge J-P, Sporns O. Can structure predict function in the human brain? Neuroimage. (2010) 52:766-76. doi: 10.1016/j.neuroimage.2010.01.071

10. Park H-J, Friston K. Structural and functional brain networks: from connections to cognition. Science. (2013) 342:1238411. doi: $10.1126 /$ science. 1238411

11. Sun Y, Yin QH, Fang R, Yan XX, Wang Y, Bezerianos A, et al. Disrupted functional brain connectivity and its association to structural connectivity in amnestic mild cognitive impairment and Alzheimer's disease. PLoS ONE. (2014) 9:14. doi: 10.1371/journal.pone.0096505

12. Wang Z, Dai Z, Gong G, Zhou C, He Y. Understanding structuralfunctional relationships in the human brain: a large-scale network perspective. Neuroscientist. (2015) 21:290-305. doi: 10.1177/1073858414537560

13. Palop JJ, Mucke L. Network abnormalities and interneuron dysfunction in Alzheimer disease. Nat Rev Neurosci. (2016) 17:777. doi: 10.1038/nrn.2016.141

14. Pereira JB, Van WD, Stomrud E, Strandberg TO, Volpe G, Westman E, et al. Abnormal structural brain connectome in individuals with preclinical Alzheimer's disease. Cereb Cortex. (2017) 13:1-12. doi: 10.1093/cercor/bhx236

15. Li D, Li T, Niu Y, Xiang J, Cao R, Liu B, et al. Reduced hemispheric asymmetry of brain anatomical networks in attention deficit hyperactivity disorder. Brain Imag Behav. (2018) 13:669-84. doi: 10.1007/s11682-018-9881-5

16. Wang B, Li T, Zhou MN, Zhao S, Niu Y, Wang X, et al. The abnormality of topological asymmetry in hemispheric brain anatomical networks in bipolar disorder. Front Neurosci. (2018) 12:12. doi: 10.3389/fnins.2018.00618

17. Wang B, Miao LW, Niu Y, Cao R, Li DD, Yan PF, et al. Abnormal functional brain networks in mild cognitive impairment and Alzheimer's disease: a minimum spanning tree analysis. J Alzheimers Dis. (2018) 65:1093-107. doi: 10.3233/JAD-180603

18. Greicius MD, Supekar K, Menon V, Dougherty RF. Resting-state functional connectivity reflects structural connectivity in the default mode network. Cereb Cortex. (2009) 19:72-8. doi: 10.1093/cercor/bhn059

19. Zhang Z, Liao W, Chen H, Mantini D, Ding J-R, Xu Q, et al. Altered functional-structural coupling of large-scale brain networks in idiopathic generalized epilepsy. Brain. (2011) 134:2912-28. doi: 10.1093/brain/awr223

20. Li K, Liu L, Yin Q, Dun W, Xu X, Liu J, et al. Abnormal rich club organization and impaired correlation between structural and functional connectivity in migraine sufferers. Brain Imag. (2017) 11:526-40. doi: 10.1007/s11682-016-9533-6

21. Dai ZJ, Lin QX, Li T, Wang X, Yuan HS, Yu X, et al. Disrupted structural and functional brain networks in Alzheimer's disease. Neurobiol. Aging. (2019) 75:71-82. doi: 10.1016/j.neurobiolaging.2018.11.005

22. Yong H, Zhang C, Gaolang G, Alan E. Neuronal networks in Alzheimer's disease. Neuroscientist. (2009) 15:333-50. doi: 10.1177/1073858409334423

23. Misra C, Fan Y, Davatzikos C. Baseline and longitudinal patterns of brain atrophy in MCI patients, and their use in prediction of short-term conversion to AD: results from ADNI. Neuroimage. (2009) 44:1415-22. doi: 10.1016/j.neuroimage.2008.10.031

24. Hahn K, Myers N, Prigarin S, Rodenacker K, Kurz A, Förstl H, et al. Selectively and progressively disrupted structural connectivity of functional brain networks in Alzheimer's disease - revealed by a novel framework to analyze edge distributions of networks detecting disruptions with strong statistical evidence. Neuroimage. (2013) 81:96-109. doi: 10.1016/j.neuroimage.2013.05.011

25. Yang L, Yan Y, Wang Y, et al. Gradual disturbances of the amplitude of lowfrequency fluctuations (ALFF) and fractional ALFF in Alzheimer spectrum[J]. Front. Neurosci. (2018) 12:975. doi: 10.3389/fnins.2018.00975
26. Yan T, Wang W, Yang L, Chen K, Chen R, Han Y. Rich club disturbances of the human connectome from subjective cognitive decline to Alzheimer's disease. Theranostics. (2018) 8:3237-55. doi: 10.7150/thno.23772

27. Wang J, Khosrowabadi R, Ng KK, Hong ZP, Chong JSX, Wang YJ, et al. Alterations in brain network topology and structural-functional connectome coupling relate to cognitive impairment. Front Aging Neurosci. (2018) 10:15. doi: 10.3389/fnagi.2018.00404

28. Qiu Y, Liu S, Hilal S, Loke YM, Ikram MK, Xu X, et al. Inter-hemispheric functional dysconnectivity mediates the association of corpus callosum degeneration with memory impairment in $\mathrm{AD}$ and amnestic MCI. Sci Rep. (2016) 6:32573. doi: 10.1038/srep32573

29. Fischer FU, Wolf D, Scheurich A, Fellgiebel A. Altered whole-brain white matter networks in preclinical Alzheimer's disease. Neuroimage Clin. (2015) 8:660-6. doi: 10.1016/j.nicl.2015.06.007

30. Shu N, Wang X, Bi Q, Zhao T, Han Y. Disrupted topologic efficiency of white matter structural connectome in individuals with subjective cognitive decline. Radiology. (2017) 286:162696. doi: 10.1148/radiol.2017162696

31. Colizza V, Flammini A, Serrano MA, Vespignani A. Detecting richclub ordering in complex networks. Nat Phys. (2006) 2:110-5. doi: $10.1038 /$ nphys209

32. Heuvel MPVD, Kahn RS, Joaquín GI, Olaf S. High-cost, high-capacity backbone for global brain communication. Proc Natl Acad Sci USA. (2012) 109:11372-7. doi: 10.1073/pnas.1203593109

33. Gareth B, Paul A, Sally Z, Nora T, Tomoki A, Nazakat M, et al. Rich-club organization of the newborn human brain. Proc Natl Acad Sci USA. (2014) 111:7456-61. doi: 10.1073/pnas.1324118111

34. Buckner RL, Jorge S, Tanveer T, Krienen FM, Hesheng L, Trey H, et al. Cortical hubs revealed by intrinsic functional connectivity: mapping, assessment of stability, and relation to Alzheimer's disease. J Neurosci. (2009) 29:1860-73. doi: 10.1523/JNEUROSCI.5062-08.2009

35. Dai Z, Yan C, Li K, Wang Z, Wang J, Cao M, et al. Identifying and mapping connectivity patterns of brain network hubs in Alzheimer's disease. Cereb Cortex. (2014) 25:3723. doi: 10.1093/cercor/bhu246

36. Daianu M, Jahanshad N, Nir TM, Jack CR Jr, Weiner MW, Bernstein MA, et al. Rich club analysis in the Alzheimer's disease connectome reveals a relatively undisturbed structural core network. Hum Brain Map. (2015) 36:3087-103. doi: $10.1002 / \mathrm{hbm} .22830$

37. Mallio CA, Schmidt R, de Reus MA, Vernieri F, Quintiliani L, Curcio $\mathrm{G}$, et al. Epicentral disruption of structural connectivity in Alzheimer's disease. CNS Neurosci Therapeut. (2015) 21:837-45. doi: 10.1111/cns. 12397

38. Zhao T, Sheng C, Bi Q, Niu W, Shu N, Han Y. Age-related differences in the topological efficiency of the brain structural connectome in amnestic mild cognitive impairment. Neurobiol Aging. 59:144-55. doi: 10.1016/j.neurobiolaging.2017.08.005

39. Morris JC. The clinical dementia rating (CDR): current version and scoring rules. Neurology. (1993) 43:2412-4. doi: 10.1212/WNL.43.11.2412-a

40. Sheik JL, Yesavage JA. Geriatric depression scale (GDS): recent evidence and develpment of shorter version. Clin Gerontol. (1986) 5:165-73. doi: 10.1300/J018v05n01_09

41. Pfeffer RI, Kurosaki TT, Harrah CH, Chance JM, Filos S. Measurement of functional activities in older adults in the community. J Gerontol. (1982) 37:323-9. doi: 10.1093/geronj/37.3.323

42. Folstein MF, Folstein SE, McHugh PR. Mini-mental state. a practical method for grading the cognitive state of patients for the clinician. J Psychiatr Res. (1975) 12:189-98. doi: 10.1016/0022-3956(75)90026-6

43. Nasreddine ZS, Phillips NA, Valérie B, Simon C, Victor W, Isabelle $\mathrm{C}$, et al. The montreal cognitive assessment, MoCA: a brief screening tool for mild cognitive impairment. J Am Geriatr Soc. (2010) 53:695-9. doi: 10.1111/j.1532-5415.2005.53221.x

44. Ghosh SS, Kakunoori S, Augustinack J, Nieto-Castanon A, Kovelman I, Gaab N, et al. Evaluating the validity of volume-based and surfacebased brain image registration for developmental cognitive neuroscience studies in children 4 to 11 years of age. Neuroimage. (2010) 53:85-93. doi: 10.1016/j.neuroimage.2010.05.075

45. Fan L, Li H, Zhuo J, Zhang Y, Wang J, Chen L, et al. The human brainnetome atlas: a new brain atlas based on connectional architecture. Cereb Cortex. (2016) 26:3508-26. doi: 10.1093/cercor/bhw157 
46. Jenkinson M, Beckmann CF, Behrens TE, Woolrich MW, Smith SM. Fsl. Neuroimage. (2012) 62:782-90. doi: 10.1016/j.neuroimage.2011.09.015

47. Cui Z, Zhong S, Xu P, He Y, Gong G. PANDA: a pipeline toolbox for analyzing brain diffusion images. Front Hum Neurosci. (2013) 7:42. doi: 10.3389/fnhum.2013.00042

48. Mori S, Crain BJ, Chacko VP, van Zijl PC. Threedimensional tracking of axonal projections in the brain by magnetic resonance imaging. Ann Neurol. (1999) 45:265-9. doi: 10.1002/1531-8249(199902)45:2<265::Aid-ana21>3.0.Co;2-3

49. Mori S, van Zijl PCM. Fiber tracking: principles and strategies - a technical review. NMR Biomed. (2002) 15:468-80. doi: 10.1002/nbm.781

50. Heuvel MPVD, Sporns O. Rich-club organization of the human connectome. J Neurosci Offic J Soc Neurosci. (2011) 31:15775-86. doi: 10.1523/JNEUROSCI.3539-11.2011

51. Yan C-G, Wang X-D, Zuo X-N, Zang Y-F. DPABI: data processing \& analysis for (resting-state) brain imaging. Neuroinformatics. (2016) 14:339-51. doi: 10.1007/s12021-016-9299-4

52. Mowinckel AM, Thomas E, Westlye LT. Network-specific effects of age and in-scanner subject motion: a resting-state fMRI study of 238 healthy adults. Neuroimage. (2012) 63:1364-73. doi: 10.1016/j.neuroimage.2012.08.004

53. Power JD, Barnes KA, Snyder AZ, Schlaggar BL, Petersen SE. Spurious but systematic correlations in functional connectivity MRI networks arise from subject motion. Neuroimage. (2012) 59:2142-54. doi: 10.1016/j.neuroimage.2011.10.018

54. Satterthwaite TD, Wolf DH, Loughead J, Ruparel K, Elliott MA, Hakonarson $\mathrm{H}$, et al. Impact of in-scanner head motion on multiple measures of functional connectivity: relevance for studies of neurodevelopment in youth. Neuroimage. (2012) 60:623-32. doi: 10.1016/j.neuroimage.2011.12.063

55. Van Dijk KR, Sabuncu MR, Buckner RL. The influence of head motion on intrinsic functional connectivity MRI. Neuroimage. (2012) 59:431-8. doi: 10.1016/j.neuroimage.2011.07.044

56. Braun U, Plichta MM, Esslinger C, Sauer C, Haddad L, Grimm O, et al. Test-retest reliability of resting-state connectivity network characteristics using fMRI and graph theoretical measures. NeuroImage. (2012) 59:1404-12. doi: 10.1016/j.neuroimage.2011.08.044

57. Fox MD, Zhang D, Snyder AZ, Raichle ME. The global signal and observed anticorrelated resting state brain networks. J Neurophysiol. (2009) 101:327083. doi: $10.1152 /$ jn. 90777.2008

58. Murphy K, Birn RM, Handwerker DA, Jones TB, Bandettini PA. The impact of global signal regression on resting state correlations: are anti-correlated networks introduced? Neuroimage. (2009) 44:893-905. doi: 10.1016/j.neuroimage.2008.09.036

59. Rubinov M, Sporns O. Complex network measures of brain connectivity: uses and interpretations. Neuroimage. (2010) 52:1059-69. doi: 10.1016/j.neuroimage.2009.10.003

60. Achard S, Bullmore ET. Efficiency and cost of economical brain functional networks. PLoS Comput Biol. (2007) 3:174-83. doi: 10.1371/journal.pcbi.0030017

61. Wang J, Wang X, Xia M, Liao X, Evans A, He Y. GRETNA: a graph theoretical network analysis toolbox for imaging connectomics. Front Hum Neurosci. (2015) 9:386. doi: 10.3389/fnhum.2015.00458

62. Xia M, Wang J, He Y. BrainNet viewer: a network visualization tool for human brain connectomics. PLoS ONE. (2013) 8:e68910. doi: 10.1371/journal.pone.0068910

63. Bi K, Hua L, Wei M, Qin J, Lu Q, Yao Z. Dynamic functionalstructural coupling within acute functional state change phases: evidence from a depression recognition study. J Affect Disord. (2016) 191:145-55. doi: 10.1016/j.jad.2015.11.041

64. Collin G, Scholtens LH, Kahn RS, Hillegers MH, van den Heuvel MP. Affected anatomical rich club and structural-functional coupling in young offspring of schizophrenia and bipolar disorder patients. Biol Psychiatr. (2017) 82:746-55. doi: 10.1016/j.biopsych.2017.06.013

65. Kim DJ, Schnakenberg Martin AM, Shin YW, Jo HJ, Cheng H, Newman SD, et al. Aberrant structural-functional coupling in adult cannabis users. Hum Brain Map. (2019) 40:252-61. doi: 10.1002/hbm. 24369

66. Jiang J, Zhou H, Duan H, Liu X, Zuo C, Huang Z, et al. A novel individuallevel morphological brain networks constructing method and its evaluation in PET and MR images. Heliyon. (2017) 3:e00475. doi: 10.1016/j.heliyon.2017. e00475

67. Collin G, Sporns O, Mandl RC, van den Heuvel MP. Structural and functional aspects relating to cost and benefit of rich club organization in the human cerebral cortex. Cereb Cortex. (2013) 24:2258-67. doi: 10.1093/cercor/bht064

68. Delbeuck X, Linden MVD, Collette F. Alzheimer' disease as a disconnection syndrome? Neuropsychol Rev. (2003) 13:79-92. doi: 10.1023/A:1023832305702

69. Dai Z, He Y. Disrupted structural and functional brain connectomes in mild cognitive impairment and Alzheimer's disease. Neurosci Bull. (2014) 30:217-32. doi: 10.1007/s12264-013-1421-0

70. Albert R, Jeong H, Barabasi AL. Error and attack tolerance of complex networks. Nature. (2000) 340:378-82. doi: 10.1038/35019019

71. Callaway DS, Newman ME, Strogatz SH, Watts DJ. Network robustness and fragility: percolation on random graphs. Phys Rev Lett. (2000) 85:5468. doi: 10.1103/PhysRevLett.85.5468

72. Frantzidis CA, Vivas AB, Tsolaki A, Klados MA, Tsolaki M, Bamidis PD. Functional disorganization of small-world brain networks in mild Alzheimer's disease and amnestic mild cognitive impairment: an EEG study using Relative Wavelet Entropy (RWE). Front Aging Neurosci. (2014) 6:224. doi: 10.3389/fnagi.2014.00224

73. Zimmermann J, Ritter P, Shen K, Rothmeier S, Schirner M, Mcintosh AR. Structural architecture supports functional organization in the human aging brain at a regionwise and network level. Hum Brain Map. (2016) 37:2645-61. doi: $10.1002 / \mathrm{hbm} .23200$

74. Sviatlana D, Aurélie BW, Louis N, Gabriel G, Armin S, Guggisberg AG. Adaptive reorganization of cortical networks in Alzheimer's disease. Clin Neurophysiol. (2013) 124:35-43. doi: 10.1016/j.clinph.2012.05.028

75. van den Heuvel MP, Mandl RCW, Kahn RS, Pol HEH. Functionally linked resting-state networks reflect the underlying structural connectivity architecture of the human brain. Hum Brain Map. (2009) 30:3127-41. doi: $10.1002 / \mathrm{hbm} .20737$

76. Vegapons S, Olivetti E, Avesani P, Dodero L, Gozzi A, Bifone A. Differential effects of brain disorders on structural and functional connectivity. Front Neurosci. (2016) 10:605. doi: 10.3389/fnins.2016.00605

77. Caeyenberghs K, Leemans A, Leunissen I, Michiels K, Swinnen SP. Topological correlations of structural and functional networks in patients with traumatic brain injury. Front Hum Neurosci. (2013) 7:726. doi: 10.3389/fnhum.2013.00726

78. Lo CY, Wang PN, Chou KH, Wang JH, He Y, Lin CP. Diffusion tensor tractography reveals abnormal topological organization in structural cortical networks in Alzheimer's disease. J Neurosci. (2010) 30:16876-85. doi: 10.1523/JNEUROSCI.4136-10.2010

79. Supekar K, Menon V, Rubin D, Musen M, Greicius MD. Network analysis of intrinsic functional brain connectivity in Alzheimer's disease. PLoS Comput Biol. (2008) 4:11. doi: 10.1371/journal.pcbi.1000100

80. Zhou YX, Dougherty JH, Hubner KF, Bai B, Cannon RL, Hutson RK. Abnormal connectivity in the posterior cingulate and hippocampus in early Alzheimer's disease and mild cognitive impairment. Alzheimers Dement. (2008) 4:265-70. doi: 10.1016/j.jalz.2008.04.006

81. Doré V, Villemagne VL, Bourgeat P, Fripp J, Acosta O, Chetélat G, et al. Cross-sectional and longitudinal analysis of the relationship between $\mathrm{a} \beta$ deposition, cortical thickness, and memory in cognitively unimpaired individuals and in Alzheimer disease. JAMA Neurol. (2013) 70:903. doi: 10.1001/jamaneurol.2013.1062

82. Blanc F, Noblet V, Philippi N, Cretin B, Foucher J, Armspach JP, et al. Right anterior insula: core region of hallucinations in cognitive neurodegenerative diseases. PLoS ONE. (2014) 9:22. doi: 10.1371/journal.pone.0114774

83. Margulies DS, Vincent JL, Kelly C, Lohmann G, Uddin LQ, Biswal BB, et al. Precuneus shares intrinsic functional architecture in humans and monkeys. Proc Natl Acad Sci USA. (2009) 106:20069-74. doi: 10.1073/pnas.09053 14106

84. Stuss DT, Alexander MP. Executive functions and the frontal lobes: a conceptual view. Psychol Res. (2000) 63:289-98. doi: 10.1007/s004269900007

85. Baddeley A. Working memory: looking back and looking forward. Nat Rev Neurosci. (2003) 4:829-39. doi: 10.1038/nrn1201

86. Bozzali M, Falini A, Franceschi M, Cercignani M, Zuffi M, Scotti G, et al. White matter damage in Alzheimer's disease assessed in vivo using diffusion 
tensor magnetic resonance imaging. J Neurol Neurosurg Psychiatr. (2002) 72:742-6. doi: 10.1136/jnnp.72.6.742

87. Choi SJ, Lim KO, Monteiro I, Reisberg B. Diffusion tensor imaging of frontal white matter microstructure in early Alzheimer's disease: a preliminary study. Geriatr Psychiatr Neurol. (2005) 18:12-9. doi: 10.1177/08919887042 71763

88. Honea RA, Swerdlow RH, Vidoni ED, Goodwin J, Burns JM. Reduced gray matter volume in normal adults with a maternal family history of Alzheimer disease. Neurology. (2010) 74:113-20. doi: 10.1212/WNL.0b013e3181c918cb

89. Horwitz B, Grady CL, Schlageter NL, Duara R, Rapoport SI. Intercorrelations of regional cerebral glucose metabolic rates in Alzheimer's disease. Brain Res. (1987) 407:294-306. doi: 10.1016/0006-8993(87)91107-3

90. Grady CL, Furey ML, Pietrini P, Horwitz B, Rapoport SI. Altered brain functional connectivity and impaired short-term memory in Alzheimer's disease. Brain A J Neurol. (2001) 124:739. doi: 10.1093/brain/124.4.739

91. Wang K, Liang M, Wang L, Tian L, Zhang X, Li K, et al. Altered functional connectivity in early Alzheimer's disease: a resting-state fMRI study. Hum Brain Map. (2010) 28:967-78. doi: 10.1002/hbm.20324
92. Hagmann P, Cammoun L, Gigandet X, Meuli R, Honey CJ, Wedeen VJ, et al. Mapping the structural core of human cerebral cortex. PLoS Biol. (2008) 6:e159. doi: 10.1371/journal.pbio.0060159

93. Li Y, Liu Y, Li J, Qin W, Li K, Yu C, et al. Brain anatomical network and intelligence. Neuroimage. (2009) 47:e1000395. doi: 10.1016/S1053-8119(09)70914-0

Conflict of Interest: The authors declare that the research was conducted in the absence of any commercial or financial relationships that could be construed as a potential conflict of interest.

Copyright (c) 2020 Cao, Wang, Gao, Li, Zhang, Hussain, Xie, Wang, Wang and Xiang. This is an open-access article distributed under the terms of the Creative Commons Attribution License (CC BY). The use, distribution or reproduction in other forums is permitted, provided the original author(s) and the copyright owner(s) are credited and that the original publication in this journal is cited, in accordance with accepted academic practice. No use, distribution or reproduction is permitted which does not comply with these terms. 